\title{
THE NATURAL LOGIC OF LANGUAGE AND COGNITION
}

\author{
Pieter A.M. Seuren
}

\begin{abstract}
This paper aims at an explanation of the discrepancies between natural intuitions and standard logic in terms of a distinction between NATURAL and CONSTRUCTED levels of cognition, applied to the way human cognition deals with sets. NATURAL SET THEORY (NST) restricts standard set theory cutting it down to naturalness. The restrictions are then translated into a theory of natural logic. The predicate logic resulting from these restrictions turns out to be that proposed in Hamilton (1860) and Jespersen (1917). Since, in this logic, NO is a quantifier in its own right, different from NOT-SOME, and given the assumption that natural lexicalization processes occur at the level of basic naturalness, single-morpheme lexicalizations for NOT-ALL should not occur, just as there is no single-morpheme lexicalization for NOT-SOME at that level. An analogous argument is developed for the systematic absence of lexicalizations for NOT-AND in propositional logic.
\end{abstract}

Keywords: Gricean maxims, Logical intuitions, Natural logic, Natural set theory, Valuation space analysis.

\section{Introductory observations}

The present study relies on the general hypothesis that there is gradient in the analytical powers of humans ranging from the "rawest" or most basic level of unsophistication to the most advanced levels of abstract thinking as found in the modern centers of science. The degree of achievement is taken to depend on the cultural and other conditions that trigger the use and development of available cognitive reserves at the right age interval. Some extremely interesting and challenging work has been done over the past few decades regarding the arithmetical capacities of infants, schoolchildren, and members of illiterate cultures. ${ }^{1}$ Pica et al. (2004) investigated the arithmetical ability of speakers of Mundurukú, an Amazonian language spoken by some 7000, mostly illiterate, Brazilian Indians. The language has no numerals beyond five (the word for 'five' being the equivalent of 'hand', as in many South-East Asian

${ }^{1}$ For example, Ginsburg et al. (1984), Dehaene (1997), Buttersworth (1999), Pica et al. (2004). 
languages), reflecting the fact that the speakers were unable to count beyond five and had great difficulty doing simple arithmetical sums. Yet after some training, they quickly extended their counting and computing abilities both cognitively and lexically, creating expressions like 'two hands', even though their achievements never matched those of humans born into culturally more developed societies (Pierre Pica, p.c.).

Butterworth states (1999: 7):

What makes human numerical ability unique is the development and transmission of cultural tools for extending the capability of the Number Module. These tools include aids to counting, such as number words, finger-counting, and tallying; and also the accumulated inventions of mathematicians down the centuries - from numerals to calculating procedures, from counting-boards to theorems and their proofs.

Unfortunately, I must disagree with Butterworth. Far from being unique to the human numerical ability, the power to extend a naturally given ability through cultural development appears to be more general. Although the exact boundaries of this human power is unknown, it does seem that, along with numeracy and, apparently, also the reading ability (Dehaene 2005) and geometrical abilities (Dehaene et al., in press), logic and its underlying set theory are worthy candidates to be included. This is what is investigated in the present paper.

NATURAL SET THEORY (NST) aims at describing the way humans deal with plural objects, standardly called "sets" in mathematical set theory. Far from assuming that the human way of dealing with sets is identical to the way they are dealt with in mathematical set theory, we assume that the latter is in many ways artificial and does not correspond to the way cognition deals with them at a natural level. NST implies a gradient cline between two extremes of cognitive achievement, which we name NATURAL SET THEORY and CONSTRUCTED SET THEORY, respectively, natural set theory manifesting itself in two forms: Either in a BASIC (or PROTOTYPICAL or DEFAULT) form or in a STRICT form, which overrides one or more defaults.

The method followed here differs from what is found in the studies mentioned above, which are experimental, performance-driven, and sometimes supported by neurophysiological evidence. Here, a hypothesis is proposed and offered for empirical testing, whereby the data are allowed to be largely intuitive. This more traditional method consists in observing how "ordinary" speakers, whose degree of literacy and cultural sophistication is considered "normal" in Western society and who distinguish themselves mainly by NOT being academically trained, interpret and use logical expressions - in particular the logical constants - in their daily speech. An attempt is then made to reduce the (well-known) differences with standard contemporary logic, which is entirely based on highly constructed mathematical set theory, to naturally given restrictions in the human way of cognitively dealing with plural sets. 
The distinction posited between what is natural and what is constructed in cognition and language embodies an attempt at making explicit the distinction between the contributions of nature and those of nurture to the cognitive structures and processes of those who participate in the advanced forms of Western culture as it has come into being over the past three or four thousand years. The distinction is hypothetical in that no experimental data are available that would support it. What follows is, therefore, tentative and exploratory, and certainly fragmentary. It fails to account, for example, for scalarity phenomena (Jespersen 1917: 82-96; Horn 1989: 204-267). Yet the distinction is intuitively appealing, and, if proved right, it will be an important element in the understanding of the logic of language. Moreover, it helps to understand certain aspects of lexicalization of logical operators, as is shown in section 5 . If it is true that universal semantic and logical properties of natural language are subject to restrictions of naturalness in a variety of ways, then, clearly, we have before us a research program of considerable magnitude - one, moreover, that has hardly been broached so far. For the moment, all we can do is present a few principles that seem to have a bearing on the matter and see how far they take us. To the extent that these first attempts are successful or inspiring, they may stimulate further research. The program thus outlined in effect amounts to an attempt at replacing current Gricean explanations in terms of the famous "maxims" for the disparity between logic and language with an explanation based on natural set theory.

\section{Some set-theoretic principles of natural cognition}

\subsection{Valuation space (VS) analysis in terms of standard theory}

A central role in the analysis is assigned to VALUATION SPACE (VS) ANALYSIS, introduced by Van Fraassen (1971). VS-analysis takes a logical language $\mathbf{L}_{\mathbf{L}}$ whose expressions, L-PROPOSITIONS, are taken to underlie the sentences of a natural language. Given one single set of reference assignments $\mathbf{K}_{\mathbf{i}}$ ("key") for all L-propositions in $\mathrm{L}_{\mathrm{L}}$, VS-analysis assigns to each $\mathrm{L}$-proposition $\mathrm{P}$ a valuation space /P/, which is the set of possible situations under $K_{\mathbf{i}}$ within the totality $\mathbf{U}$ of all possible situations in which $P$ is true. A POSSIBLE SITUATION is defined as a set of truth-value assignments under any given $\mathbf{K}_{\mathbf{i}}$ to all L-propositions of $\mathbf{L}_{\mathbf{L}}$. If $\mathbf{L}_{\mathbf{L}}$ contains $n$ logically and semantically independent L-propositions, each of which allows for truth $(\mathrm{T})$ or falsity $(\mathrm{F})$ independently of all the others, then, in a standard bivalent system of truth and falsity, there are, under each $\mathbf{K}_{\mathbf{i}}, 2^{\mathrm{n}}$ logically and semantically independent valuations, that is, assignments of $\mathrm{T}$ or $F$ to each of the L-propositions of the language. Each such set of assignments is called a VALUATION or POSSIBLE SITUATION. Given a particular L-proposition P, the VALUATION SPACE of $P$, written as $/ P /$, is the set of possible situations 
(valuations), under any given $\mathbf{K}_{\mathbf{i}}$, in which $\mathrm{P}$ is assigned the value T. An Lproposition $\mathrm{P}$ is true just in case the actual situation $s_{a c t}$ is an element in $/ \mathrm{P} /$. Clearly, L-propositions formed with the help of one or more truth-functional propositional operators have their truth value assigned depending on the truthvalues of the component L-proposition(s) and the way the truth function is defined. Similar restrictions hold for relations of entailment or contrariety depending on nonformalized semantic conditions.

Although it would be utterly unrealistic to assume that natural speakers have a fully elaborated interpretation of set theory onto a system of valuation spaces at their disposal when interpreting utterances, it is not at all unrealistic to assume that they have an ill-defined, intuitive idea, probably beyond the threshold of possible awareness, of "truth in an overall, possibly infinite, set of situations," and hence of notions like necessary consequence (in)compatibility, and contradiction. Correspondingly, one may assume that notions like mutual exclusion, partial intersection, and proper inclusion of sets of situations are likely to be psychologically real, these notions being defined without any appeal to the extreme boundaries of the set-theoretic system, namely the null set $\varnothing$ and the totality of all objects OBJ. Since mutual exclusion, partial intersection, and proper inclusion are the key notions in both NST and its application to natural logic, it seems reasonable to assume psychological reality for both NST and natural logic.

The instrument of VS-analysis turns out to be of great use in logical analysis, if only because it enables one to establish a very useful link between Boolean algebra and propositional logic, different from the standard link, which was established by Frege. In the standard Fregean view, propositional logic is a specific interpretation of Boolean algebra with 1 and 0 , interpreted as TRUE and FALSE, respectively, as the only values for the variables: No other constants are allowed (hence the convention of designating truth by 1 and falsity by 0 ). Propositions of the logical language are designated by their truth values, taken to be their extensions. The propositional truth functions are now computed according to the Boolean operations, which give the standard truth tables.

Negation corresponds to Boolean complement:

$$
1,-=0 \text { and } 0,-=1 .
$$

Conjunction $(\wedge)$ corresponds to Boolean multiplication $(\bullet)$ :

$$
1 \cdot 1=1 ; 1 \bullet 0=0 ; 0 \bullet 1=0 ; 0 \bullet 0=0 .
$$

Disjunction $(\vee)$ corresponds to Boolean addition $(+)$ :

$$
1+1=1 ; 1+0=1 ; 0+1=1 ; 0+0=0 .
$$

VS-analysis allows for a different interpretation of Boolean algebra onto propositional logic, in that the set-theoretic functions are used as an intermediary. First, we use the standard interpretation of Boolean algebra onto set theory, which treats 1 as the universe $\mathbf{O B} \mathbf{J}$ of all objects and 0 as the null set $\varnothing$. The Boolean variables are taken to range over sets, so that set-theoretic COMPLEMENT corresponds to Boolean complement, set-theoretic INTERSECTION 
to Boolean multiplication, and set-theoretic UNION to Boolean addition. This translates directly onto the truth functions of propositional logic, with VSs as sets and the universe $\mathbf{U}$ of all possible situations as $\mathbf{O B J}$, in the following way:

Negation corresponds to set-theoretic complement:

for any L-proposition $\mathrm{P}: / \neg \mathrm{P} /=/ \mathrm{P} /,-$

Conjunction $(\wedge)$ corresponds to set-theoretic intersection $(\cap)$ :

for any L-propositions $P$ and $Q: / P \wedge Q /=/ P / \cap / Q /$.

Disjunction $(\vee)$ corresponds to to set-theoretic union $(\cup)$ :

for any L-propositions $P$ and $Q: / P \vee Q /=/ P / \cup / Q /$.

This allows one to treat the logical truth functions as predicates in the following way:

Negation: $\neg \mathrm{P}$ is true iff $\quad$ the actual situation $s_{a c t} \in / \mathrm{P} /$,

Conjunction: $P \wedge Q$ is true iff the actual situation $s_{a c t} \in / P / \cap / Q /$.

Disjunction: $P \vee Q$ is true iff the actual situation $s_{a c t} \in / P / \cup / Q /$.

Moreover, the metalogical relations are now expressible as set-theoretic VSrelations. Logical equivalence $(\equiv)$ corresponds to set-theoretic identity $(=)$, entailment $(I,-)$ to inclusion, contradictoriness (CD) to mutual exclusion and exhaustion of $\mathbf{U}$ (for all $s \in \mathbf{U}, \mathrm{s} \in / \mathrm{P} /$ or $\mathrm{s} \in / \mathrm{Q} /$; called FULL UNION below), contrariety $(\mathrm{C})$ to mutual exclusion, and subcontrariety (SC) to exhaustion of $\mathbf{U}$, as shown in (1):

(1) For any L-propositions $P$ and $Q$ :
a. $\quad \mathrm{P} \equiv \mathrm{Q}) \quad$ iff $/ \mathrm{P} /=/ \mathrm{Q} /$
b. $P I,-Q \quad$ iff $/ P / \subseteq / Q /$
c. $\mathrm{CD}(\mathrm{P}, \mathrm{Q})$ iff $/ \mathrm{P} / \cup / \mathrm{Q} /=_{\mathrm{V}} \mathbf{U}$ and $/ \mathrm{P} / \cap / \mathrm{Q} /=_{\mathrm{V}} \varnothing$ (or: $/ \mathrm{P} /=/ \mathrm{Q} /,-$ )
d. $C(P, Q)$ iff $/ P / \cap / Q /={ }_{V} \varnothing$
e. $\mathrm{SC}(\mathrm{P}, \mathrm{Q})$ iff $/ \mathrm{P} / \cup / \mathrm{Q} /=_{\mathrm{V}} \mathrm{U}$

VS-analysis greatly facilitates simple diagrammatic representations which show immediately the essential properties of logical systems, without lengthy proofs.

It is important to emphasize the distinction between those set-theoretic functions that map $n$-tuples of sets onto sets and those that map $n$-tuples of sets onto truth values. The former are usually just called SET-THEORETIC FUNCTIONS; the latter are often called RELATIONS, but they may as well be called PREDICATES, since predicates are typically functions from $n$-tuples of objects, or sets of objects, to truth values. COMPLEMENT, INTERSECTION, UNION, and SUBTRACTION are set-theoretic functions, but INCLUSION, for example, is a relation: For any given sets $A$ and $B$, the binary relation of INCLUSION, as in $A \subseteq$ $B$, is either true or false. The set functions $A,{ }^{-}, A \cap B, A \cup B$, or $A-B$ do not have a truth value. Given the proper number of arbitrary sets (one for complement; more than one for intersection and union; exactly two for subtraction) they 
denote a new set defined by the Boolean functions COMPLEMENT, INTERSECTION, UNION, and SUBTRACTION, respectively. In logic, a further use of the term COMPLEMENT is to denote a relation yielding truth, for any given sets $A$ and $B$, just in case $\mathrm{A} \cup \mathrm{B}=\mathbf{O B} \mathbf{J}$ and $\mathrm{A} \cap \mathrm{B}=\varnothing$.

The set-theoretic RELATIONS have a twofold use in the reduction of logic to set theory. First, they correspond to metalogical relations expressed in terms of VS-analysis. For example, the inclusion relation translates into a possible metalogical statement that, say, the set of situations / $P$ / is included in the set of situations / $Q$ / - that is, $P$ entails $Q(P I,-Q)$ - which is true or false depending on the meanings of $P$ and $Q$, respectively, including the meanings of the truthfunctional propositional operators. Secondly, some set-theoretic relations correspond to QUANTIFIERS. For example, when I say All $F$ is $G$, this translates, in principle, as saying that the set $A$ is included in the set $B$ - following the theory of generalized quantifiers (Barwise \& Cooper 1981).

By contrast, the set-theoretic FUNCTIONS correspond to the propositional logical operators (constants) of the object language $\mathbf{L}_{\mathbf{L}}$. Just as the set-theoretic functions take sets and deliver sets, the propositional functions take valuation spaces and deliver valuation spaces. For example, the operator and in an Lproposition of the form $P$ and $Q$ delivers / $P$ and $Q /$ - that is, the set of those situations that make $P$ and $Q$ true, corresponding to $/ P / \cap / Q /$, the intersection of $/ P /$ and /Q/. Figure 1 shows how set-theoretic relations and functions are interpreted onto metalogical relations, object-language quantifiers, and propositional operators.

The counterpart in metalogic of the set-theoretic relation PARTIAL INTERSECTION (the two sets partially intersect and do not severally or jointly equal either $\mathbf{U}$ or $\varnothing$, as in Figure $3-b$ ) is LOGICO-SEMANTIC INDEPENDENCE, which plays no part in the machinery of logic: When /P/ and /Q/ partially intersect, the L-propositions $P$ and $Q$ are logically (and semantically) independent in the sense that the actual situation $s_{\text {act }}$ can be in $/ \mathrm{P} /$ but not in /Q/, in /Q/ but not in / $/$ /, in both / $/$ and /Q/, or in neither / $/$ nor / $/$. That is, no entailment relation whatsoever holds between $P$ and $Q$.

Moreover, the relations COMPLEMENT, IDENTITY, and FULL UNION (union such that the union of the sets involved exhausts OBJ; introduced because it corresponds to subcontrariety in traditional logic) lack a quantificational counterpart in predicate calculus, perhaps because they lack logical interest or perhaps because the makers of modern logic did not see far enough beyond natural language. Finally, the set-theoretic function SUBTRACTION lacks a singlemorpheme propositional counterpart, at least in most languages, but it is expressed as and/but not: The valuation space of an L-proposition of the form $P$ but not $Q$ is the VS of $P$ minus the VS of $Q$, or $/ P /-/ Q /$. 


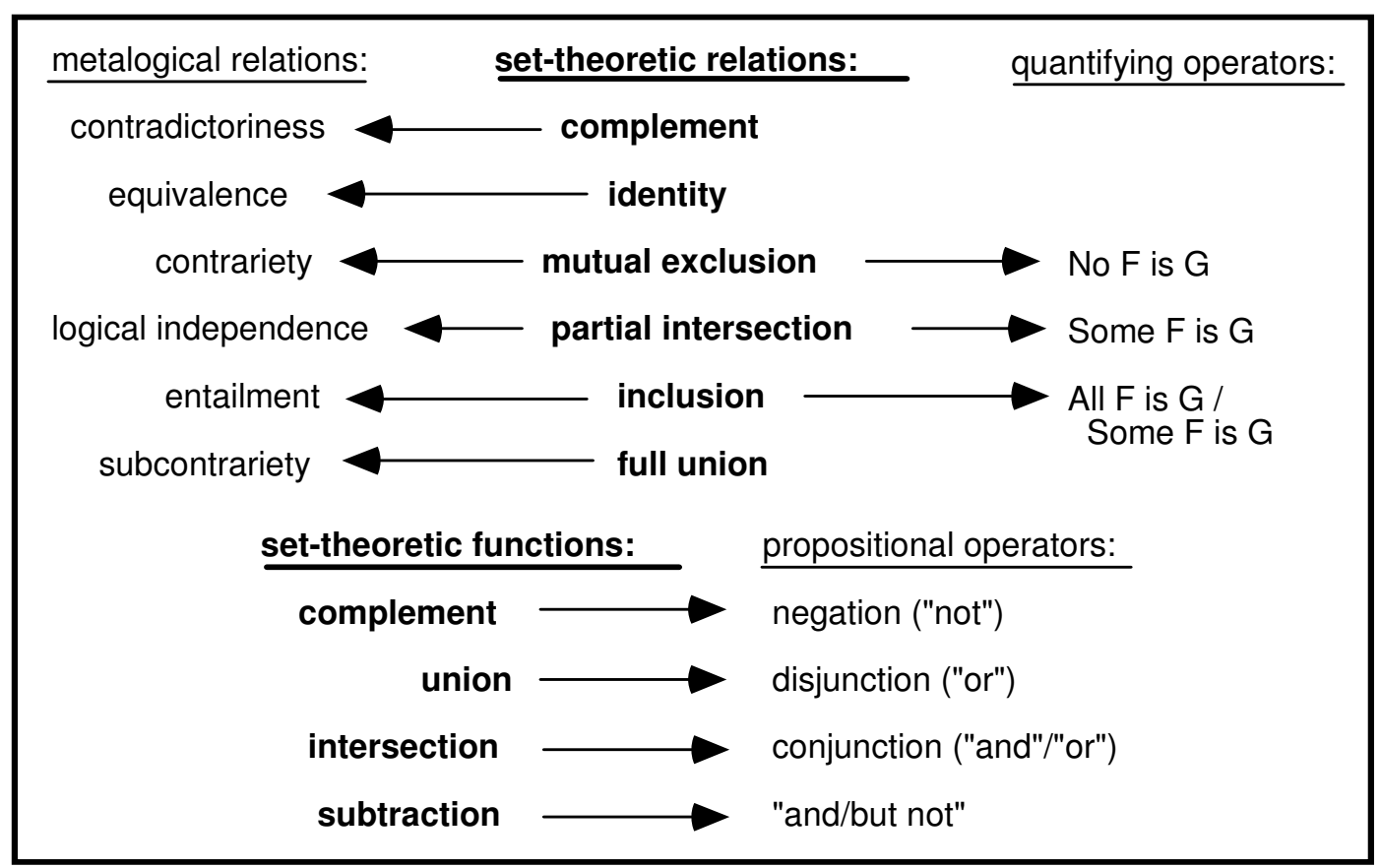

Figure 1 The reduction of metalogical relations and object-language operators to set-theoretic relations and functions

\subsection{The restrictions imposed by NST}

So much for standard set theory and standard logic. The question is now: What restrictions are to be imposed on this system so that the discrepancies between logic and language are correctly predicted? To begin with, let it be assumed that NST entails that the mind does not naturally represent a set as either the null set $(\varnothing)$ or the universe of all objects (OBJ). In NST, the null set is not a set at all: the cognitive counterpart of $\varnothing$ is the absence of any set, something which is cognitively real and may be called "null" but cannot play the role of a set. Whereas "null" still functions cognitively as "absence of a set," the opposite notion of OBJ is typically the product of advanced mathematical and/or philosophical construction. OBJ, as an abstract notion in standard modern settheoretic thinking, is taken to be too nondescript to be cognitively real and thus to play no role in natural set theory. What does seem to play a role in it is the notion of a RESTRICTED UNIVERSE OF OBJECTS or OBJ $\mathbf{R}$, involving the totality of all objects within a contextually defined universe of discourse. Therefore, all standard set-theoretic definitions involving OBJ should, for the purpose of natural set theory, be redefined as involving the notion of OBJR, which does count as a natural set.

Given these assumptions, we posit the first PRINCIPLE OF NATURAL SET THEORY, PNST-1, which applies to single sets: 


\section{PNST-1: $\quad \varnothing$ AND OBJ ARE NOT NATURAL SETS}

Sets are never cognitively represented as having an EXTREME VALUE - that is, as the null set $(\varnothing)$ or as the totality of objects (OBJ).

Sets that fulfill the condition of PNST-1 are called NATURAL SETS. In the absence of any experimental data, and hence of any precise scale of naturalness, we posit, more or less intuitively, that PNST-1 is both basically and strictly natural in that it strongly resists intellectual construction. Only at a very advanced level will the cognitive powers of the human race be able to override PNST-1.

One notes that it does not seem to matter, for natural cognition, whether a set is finite or, technically speaking, infinite, as the notion "very large" appears to cover both "infinite" and "very large but finite."

A further principle of natural set theory requires the nonidentity, or distinctness, of any two or more natural set representations. When two or more sets are cognitively represented, they are naturally taken to be DISTINCT from each other: Any relation of identity is considered constructed. This gives the second principle of natural set theory, which eliminates the RELATION OF IDENTITY:

\section{PNST-2: NATURAL SETS ARE DISTINCT}

When sets are distinguished cognitively, they are represented as being DISTINCT as regards their membership. There is no natural cognitive relation of identity between sets.

Like PNST-1, PNST-2 is taken to be natural in both a basic and a strict sense, in that it takes a considerable amount of advanced analytical thinking - much more than natural cognition and common natural language can bear - for it to be overridden.

In actual fact, PNST-2 is more general. It applies to any kind of object representation, not only of sets but also of individual objects: No two objects of any kind allow for a RELATION OF IDENTITY, since that would make them one single object. Yet they do allow for a mental OPERATION OF IDENTIFICATION, given distinct levels of representation. This is what underlies the predicate of identity in natural language: What was thought to be distinct becomes one. Such an operation involves two levels of cognitive representation, one at which the two virtual objects or sets are distinct and one at which they have merged into one. If truth is claimed for the latter, two virtual objects or sets have been identified. If truth is claimed for the former, one virtual object or set has been cognitively split up into two.

Moreover, the identity predicate, which identifies as one what were thought to be different objects, is to be distinguished from the value-assigning predicate $b e_{v}$, which assigns a value to a parameter, as in My name is Pieter, where my 
name denotes the parameter and Pieter denotes the value assigned to the parameter. Obviously, the value-assigning predicate $b e_{v}$ cannot be missed in a system consisting of functions. Therefore, $b e_{v}$ is admitted, allowing for free substitution of either of its terms in any context. In standard Boolean and settheoretic expressions we will henceforth distinguish between the identity predicate $(=)$ and the value-assigning predicate $b e_{v}$ written as $=_{\mathrm{v}}\left(\right.$ or ${ }_{\mathrm{v}}=$ when the value precedes the function).

We assume, furthermore, that PNST-2 has three subprinciples, all three less basic than PNST-1 and PNST-2, so that they can be overridden at the level of strict naturalness. They apply to the set-theoretic functions of UNION, SUBTRACTION, and INTERSECTION, respectively ( $|X|$ stands for the cardinality of any set $\mathrm{X})$ :

\section{PNST-2a: BASIC NATURAL UNION REQUIRES TOTAL DISTINCTNESS}

When two (or more) naturally represented sets $A$ and $B$ are added by the function UNION, they are, at the level of basic naturalness, TOTALLY DISTINCT, without any element in common, so that $|\mathrm{A} \cup \mathrm{B}|=|\mathrm{A}|+|\mathrm{B}|$.

Under PNST-2a, union is thus defined, at the level of basic naturalness, only for any two (or more) totally distinct natural sets. When this condition is not fulfilled, there is, at that basic level, no union.

PNST-2b is the obverse of PNST-2a, in that it requires total (proper) inclusion for the basic natural function of subtraction:

\section{PNST-2b: BASIC NATURAL SUBTRACTION REQUIRES PROPER INCLUSION}

At the level of basic naturalness, when a set $A$ is subtracted from a set $B$ by

SUBTRACTION, $A$ is a proper subset of $B$, so that $|\mathrm{B}-\mathrm{A}|=|\mathrm{B}|-|\mathrm{A}|$.

Under PNST-2b, subtraction is defined, at the level of basic naturalness, for any two natural sets $A$ and $B$ such that, if $A$ is subtracted from $B, A$ is a proper subset of $B$. When this condition is not fulfilled, there is no subtraction, at that level.

The conditions expressed in these two subprinciples have been axiomatized in standard arithmetic for the calculus of cardinality. They ensure that, under the principles of natural set theory, Boolean addition and subtraction agree with their arithmetical namesakes as regards the cardinality of the sets involved.

By contrast, the set-theoretic function INTERSECTION, and its Boolean counterpart MULTIPLICATION, do not correspond to any arithmetical function. PNST-2c defines the natural intersection function as excluding both total distinctness and proper inclusion, leaving only partial intersection: 
PNST-2c: BASIC NATURAL INTERSECTION IS PARTIAL

At the level of basic naturalness, the function INTERSECTION of two sets $A$ and $B$ requires that $A \cap B$ be nonnull (also for strict naturalness) and be properly included in both $A$ and $B$ (only for basic naturalness).

PNST-2c thus defines, at the level of basic naturalness, intersection only for those sets $A$ and $B$ (and possibly more) that satisfy the condition of actually intersecting $(A \cap B \bullet \varnothing)$, whereby the extra condition holds that the intersection be properly included in both ( $\mathrm{A} \cap \mathrm{B} \subset \mathrm{A}$ and $\mathrm{A} \cap \mathrm{B} \subset \mathrm{B}$ ). Yet, while PNST-2c is absolute-that is, both basically and strictly natural - for the condition that $\mathrm{A} \cap \mathrm{B} \bullet \varnothing$, it appears to be merely basically natural, and hence relatively easily overridden, for the condition that $A \cap B \subset A$ and $A \cap B \subset B$, creating the possibility that $A \cap B={ }_{v} A$ or $A \cap B={ }_{v} B$. (This easy overriding allows for the upgrading of exclusive to inclusive or and for the upgrading of some excluding all to some including all, as is shown in section 4 , and hence for the subaltern entailment schema from All F is $G$ to Some $F$ is G.)

Thus restricted, the natural intersection function has the unique property of ensuring SET-THEORETIC INDEPENDENCE. That is, if $\mathrm{A} \cap \mathrm{B}$ has a value under PNST-2c, then $A$ and $B$ are set-theoretically independent, and vice versa. It is then ensured that, for any element $o \in \mathbf{O B J}$, it is possible that $o \in \mathrm{A}$ and $o \notin \mathrm{B}$, or $o \in \mathrm{B}$ and $o \notin \mathrm{A}$, or $o \in \mathrm{A}$ and $o \in \mathrm{B}$, or $o \notin \mathrm{A}$ and $o \notin \mathrm{B}$.

There is a final principle, relating to the recursive application of the settheoretic functions. Whereas in mathematical set-theory one can apply the functions recursively to one's heart's content, this is not so in natural set theory. This applies especially to the function COMPLEMENT, which is nonrecursive in basic NST:

\section{PNST-3: BASIC COMPLEMENT IS NONRECURSIVE}

At the level of basic naturalness, COMPLEMENT is restricted to one application. Double complement is admitted in strictly natural set theory; further applications are constructed.

Psychological experiments should provide greater clarity and greater precision. 


\section{Consequences for set-theoretic and logical relations and functions}

\subsection{Consequences for set-theoretic relations and functions}

Formally, basic NST amounts to an application of Boolean algebra, whereby the restrictions as formulated in $(2 \mathrm{a}-\mathrm{f})$ hold. In $(2 \mathrm{a}-\mathrm{f})$ a notational distinction is made between the standard Boolean operators and their basic natural counterparts. The latter are provided with the superscript $\mathrm{BN}$ (basic natural). One notes that the definitions of the $\mathrm{BN}$-operators require an appeal to the standard operators.

(2) a. PNST-1: $\quad 0$ and 1 are excluded as values of the Boolean variables.

b. PNST-2: the relation $=(\bullet)$ is eliminated; only the value-assigning relation $=_{\mathrm{v}}$ $\left(\right.$ or $_{\mathrm{V}}=$ ) and its negative counterpart $\bullet_{\mathrm{V}}\left(\right.$ or $_{\mathrm{V}} \bullet$ ) are admitted.

c. PNST-2a: ADDITION $\left(\mathrm{x}+{ }^{\mathrm{BN}} \mathrm{y}\right)$ is restricted to $x, y$ such that there is no $z$ such that $x \bullet y={ }_{v} z$.

d. PNST-2b: SUBTRACTION $(x-B N y)$ is restricted to $x, y$ such that

$$
\begin{aligned}
& x \bullet y={ }_{v} y \\
& x \bullet y{ }_{v} x .
\end{aligned}
$$

e. PNST-2c: MULTIPLICATION $\left(x \bullet \mathrm{BN}_{\mathrm{y}}\right)$ is restricted to $x, y$ such that

$$
\mathrm{x} \bullet \mathrm{y} \cdot{ }_{\mathrm{v}} \mathrm{x} \bullet \mathrm{v} \mathrm{y} \text {. }
$$

there is a $z$ such that $x \bullet y={ }_{v} z$

f. PNST-3: COMPLEMENT $\left(\mathrm{x}^{-}{ }^{-\mathrm{BN}}\right)$ is non-recursive and restricted to an independently given element $y$ in the range of the variables such that there is a $z$ such that $y-\mathrm{BN}_{\mathrm{X}}={ }_{\mathrm{V}} \mathrm{z}$. (We say: " $\mathrm{x}$, - in $\mathrm{y}={ }_{\mathrm{V}} \quad z^{\prime}$.)

Thus restricted, Boolean algebra is translatable into basic NST as follows:

(3) a. $\cdot \mathrm{BN}$ is interpreted as basic natural intersection $\cap \mathrm{BN}$.

b. $+{ }^{\mathrm{BN}}$ is interpreted as basic natural union $\cup \mathrm{BN}$.

c. $\mathrm{x}^{-} \mathrm{BN}$ is interpreted as restricted complement $\mathrm{X}^{-\mathrm{R}}$.

d. $-B N$ is interpreted as basic natural subtraction $\_$BN.

This, in its turn, is interpretable onto a logical system when the logical constants are defined in set-theoretic terms. In anticipation of the results of section 4.1, we say that the resulting predicate logic, shown in Figure 9 below, is the (faulty) logic proposed in Hamilton (1860) and Jespersen (1917). ALL, SOME, and $\mathrm{NO}$ are defined in $(5 \mathrm{a}-\mathrm{c})$, where $\mathbf{A}$ stands for All $\mathrm{F}$ is $\mathrm{G}, \mathbf{I}$ for Some $F$ is $\mathbf{G}$, 
and $\mathbf{E}$ for No F is G. For internal or "subsentential" negation, as in All/some/no F is not $G$, [[G] is to be replaced with [[G]], ${ }^{--}$, the restricted complement of [[G]] in any presuppositionally restricted universe of objects. The external negations of sentences with ALL, SOME, and NO are defined in (5d-f). The sign $\neg$ for the external negation in the logical language $\mathbf{L}_{\mathbf{L}}$ is replaced with $\sim$, which selects the complement in the restricted universe of all possible situations $\mathbf{U} \mathbf{R}$. One notes that $(5 c, e)$ show that $\sim \mathbf{I}$ does not equal $\mathbf{E}$, since the conditions of $\mathbf{E}$ exclude [[F]] $\subset[[G]]$, whereas those of $\sim$ I allow for $[[F]] \subset[[G]]$ (that is, when $s_{\text {act }} \in / \mathbf{A} /$ ). One also notes that basic natural proper inclusion $(\subset)$ does not differ from its standard counterpart. In terms of NST it is defined as follows: $\mathrm{A} \subset \mathrm{B}$ is true iff there is a set $\mathrm{C}$ such that $\mathrm{B}-\mathrm{BN} \mathrm{A}={ }_{\mathrm{V}} \mathrm{C}$.

\begin{tabular}{|c|c|c|c|}
\hline (5) a. & ALL F IS G (A) & is true iff & {$[[\mathrm{F}]] \subset[[\mathrm{G}]]$} \\
\hline b. & SOME F IS G (I) & is true iff & there is a set $\mathrm{H}$ such that $\mathrm{H} \subset[[\mathrm{F}]]$ and $\mathrm{H} \subset[[\mathrm{G}]]$ \\
\hline c. & NO F IS G (E) & is true iff & $\begin{array}{l}\text { there is no set } \mathrm{H} \text { such that }[[\mathrm{F}]] \cap \cap^{\mathrm{BN}}[[\mathrm{G}]]={ }_{\mathrm{V}} \mathrm{H} \text {; } \\
{[[\mathrm{F}]] \not \subset[[\mathrm{G}]] ;[[\mathrm{G}]] \not \subset[[\mathrm{F}]]}\end{array}$ \\
\hline d. & $\sim \mathbf{A}$ & is true iff & $s_{a c t} \in / \mathbf{A} /,-\mathbf{R}_{\text {in }} \mathbf{U} \mathbf{R} s_{a c t} \in / \mathbf{I} /$ or $/ \mathbf{E} /$ or both \\
\hline e. & $\sim 1$ & is true iff & $s_{a c t} \in / \mathbf{I} /,-\mathbf{R}_{\text {in }} \mathbf{U} \mathbf{R}_{:} s_{a c t} \in / \mathbf{A} /$ or $/ \mathbf{E} /$ or both \\
\hline f. & $\sim E$ & is true iff & $s_{a c t} \in / \mathbf{E} /,-\mathbf{R}_{\text {in }} \mathbf{U}^{\mathbf{R}}: s_{a c t} \in / \mathbf{A} /$ or $/ \mathbf{I} /$ or both. \\
\hline
\end{tabular}

The VS-model of Figure 9, which shows the basic natural HamiltonJespersen system of quantification, is a direct consequence of the definitions in (5a-f). Its propositional counterpart, shown in Figure 10, results from reading AND as expressing $\cap^{\mathrm{BN}}$, OR as expressing $\cup^{\mathrm{BN}}, \sim$ as expressing $X^{-}{ }^{-}$, and NOR as expressing the intersection of / AND/ and / OR/ in Figure 10 (subtraction plays no role).

The formal sketch given in (2) and (3) requires some comment. We start with RESTRICTED COMPLEMENT as a function and as a relation between a set $\mathrm{A}$ and its complement $A,{ }^{-}$. Neither the function nor the relation are current in standard set theory, yet they are of central importance to the study of natural cognition and natural language. The FUNCTION, written as $X^{-}{ }^{-R}$, is defined in (6a): it takes OBJ $\mathbf{R}$ as given in any situation and any set $X \subset \mathbf{O B J} \mathbf{R}$, and it delivers $\mathbf{O B} \mathbf{J} \mathbf{R}_{-} \mathbf{X}$. The corresponding RELATION RC between a set $X$ and its restricted complement $Y$ within $\mathbf{O B J}^{\mathbf{R}}$ is defined in (6b):

(6) a. $\quad X^{-R}=\operatorname{def}$ the set $Y$ such that $Y_{V}=O_{B} \mathbf{R}{ }_{-} B N X$.

b. RESTRICTED COMPLEMENT: $\mathbf{R C}\left(Y_{,}, \mathbf{O B} \mathbf{J} \mathbf{R}\right)$ iff $X \subset \mathbf{O B J} \mathbf{R}$ and $\mathrm{Y}_{\mathrm{V}}=\mathbf{O B J} \mathbf{R}-\mathrm{X}$. 
Thus, in Figure 2, A, ${ }^{-R}$ equals OBJ $\mathbf{R}_{-} \mathrm{BN}$ A (but remember that multiple applications of this function are excluded in virtue of PNST-3). The relation between $\mathrm{A}$ and $\mathrm{A},{ }^{-\mathrm{R}}$ (horizontal lines) corresponds to natural contradictoriness (that is, within the restricted complement); that between $A$ and $\mathrm{A}^{-}$(vertical lines) to the standard metalogical relation of that name.

The set-theoretic relation of IDENTITY has been eliminated in virtue of PNST2, as has been said. FULL UNION is likewise eliminated, at least for basic naturalness: When $A$ and $B$ intersect it is eliminated in virtue of PNST-2a; when they do not, full union equals complement. More is said about full union in a moment.

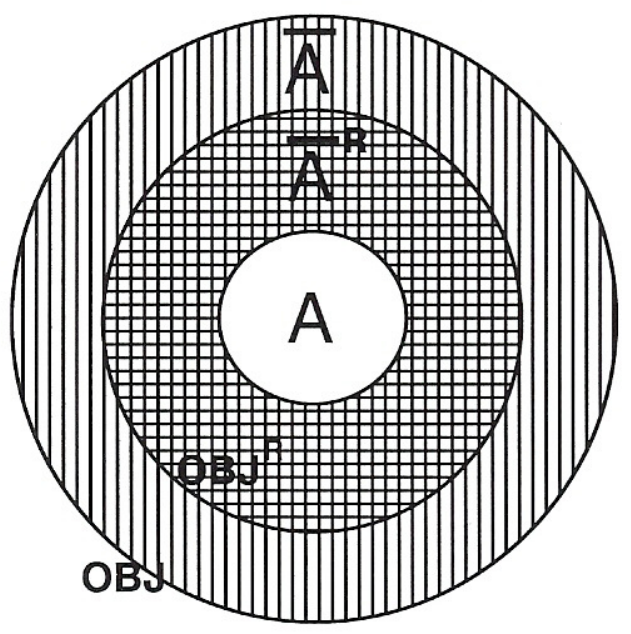

Figure 2 The relation between the natural set $\mathrm{A}$, its restricted complement $\mathrm{A},{ }^{-\mathrm{R}}$, and its standard complement $\mathrm{A}^{-}$

The result is that, while $\mathbf{O B J}$ is replaced with $\mathbf{O B J \mathbf { R }}$, there are only three possible basic natural relations left between any two natural sets $A$ and $B$ : MUTUAL EXCLUSION, PARTIAL INTERSECTION, and PROPER INCLUSION. They are defined, in standard terms, in $(7 a, b, c)$ and shown in Figure $3-a, b, c$, respectively:

(7) Basic natural relations between natural sets $A$ and $B$ within $\mathbf{O B J} \mathbf{R}$ :
a. MUTUAL EXCLUSION
iff $A \cap B={ }_{v} \varnothing$
b. PARTIAL INTERSECTION
iff $A \cap B \cdot \bullet_{V} \varnothing$
$\mathrm{A} \cap \mathrm{B},{ }^{-\mathrm{R}} \cdot{ }_{\mathrm{V}} \varnothing$
$B \cap A,{ }^{-R} \cdot v \varnothing$
c. INCLUSION of $A$ in $B$
iff $\mathrm{B}-\mathrm{A} \cdot \bullet_{\mathrm{V}} \varnothing$
$A \cap B,{ }^{-R}={ }_{V} \varnothing$



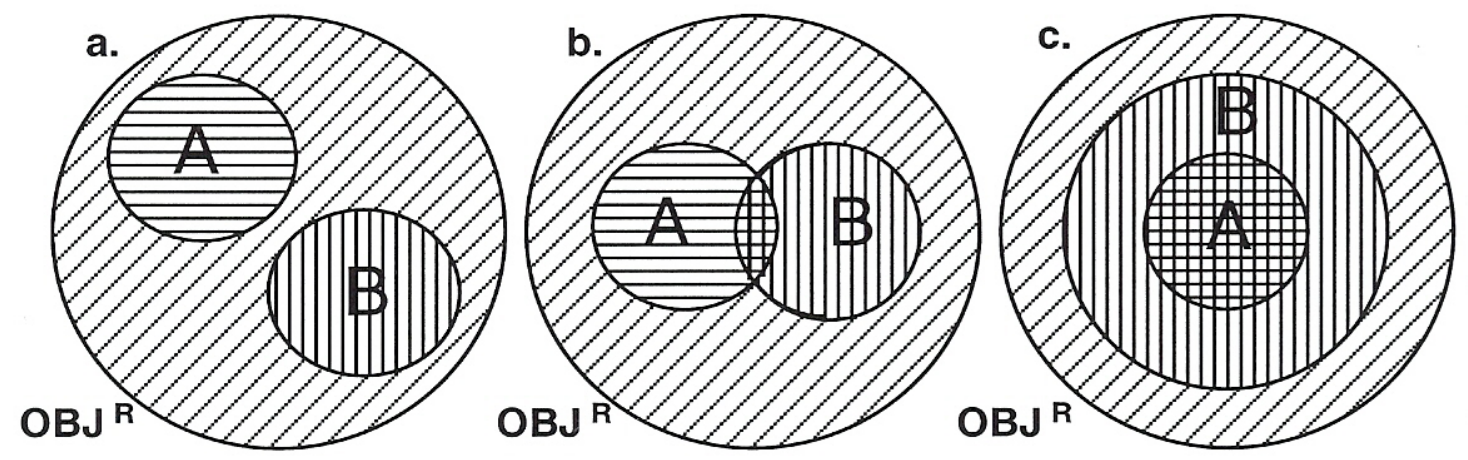

Figure 3 MUTUAL EXCLUSION, PARTIAL INTERSECTION, and PROPER

INCLUSION as the three basic natural relations between sets $A$ and $B$

INCLUSION has now been reduced to PROPER INCLUSION, as the difference hinges on the identity of A and B. This is precisely what is needed, since the inclusion relation strikes nonmathematicians as nonnatural for two identical sets. Moreover, INTERSECTION has been reduced to PARTIAL INTERSECTION because when, in standard terms, $A \cap B={ }_{v} \varnothing$, there is no intersection, and when $\mathrm{A} \cap \mathrm{B}={ }_{\mathrm{v}} \mathrm{A}$ or $\mathrm{A} \cap \mathrm{B}={ }_{\mathrm{v}} \mathrm{B}$, there is (proper) inclusion but not intersection, according to PNST-2c (though, as has been said, this latter condition appears to apply only to basic, not to strict, naturalness).

MUTUAL EXCLUSION has passed relatively unscathed through the naturalness restrictions. In particular, it still allows for $A$ and $A^{-}{ }^{-}$, as in Figure 2, to be called mutually exclusive. What makes Figure 2 a special case of mutual exclusion is that the union of $A$ and $A,{ }^{-R}$ exhausts $\mathbf{O B J} \mathbf{R}$, or $A \cup A^{-}{ }^{-R}={ }_{V}$ OBJR.

The same freedom for the union of $A$ and $B$ to exhaust $\mathbf{O B} \mathbf{J}^{\mathbf{R}}$ is, however, not granted to them when they partially intersect, as in Figure 4-a, or when the one is (properly) included in the other, as in Figure 4-b. In those cases it may be true in standard terms that $\mathrm{A} \cup \mathrm{B}={ }_{\mathrm{v}}$ OBJR, but it is not true under the restriction imposed by PNST-2a, because PNST-2a leaves basic natural union undefined for cases where $A$ and $B$ are not totally distinct. FULL UNION defined here as (8) in a form adapted to NST, is thus equally undefined when $A$ and $B$ are not totally distinct, even when $\mathbf{O B J}$ is replaced with $\mathbf{O B J} \mathbf{R}^{\text {("FUR" stands }}$ for full union within any given $\mathbf{O B J} \mathbf{R}$ ).

$$
\mathbf{F U}^{\mathbf{R}_{(}}(\mathrm{A}, \mathrm{B}) \text { iff } \mathrm{A} \cup \cup^{\mathrm{BN}} \mathrm{B}_{{ }_{\mathrm{V}}} \text { OBJ } \mathbf{R}
$$



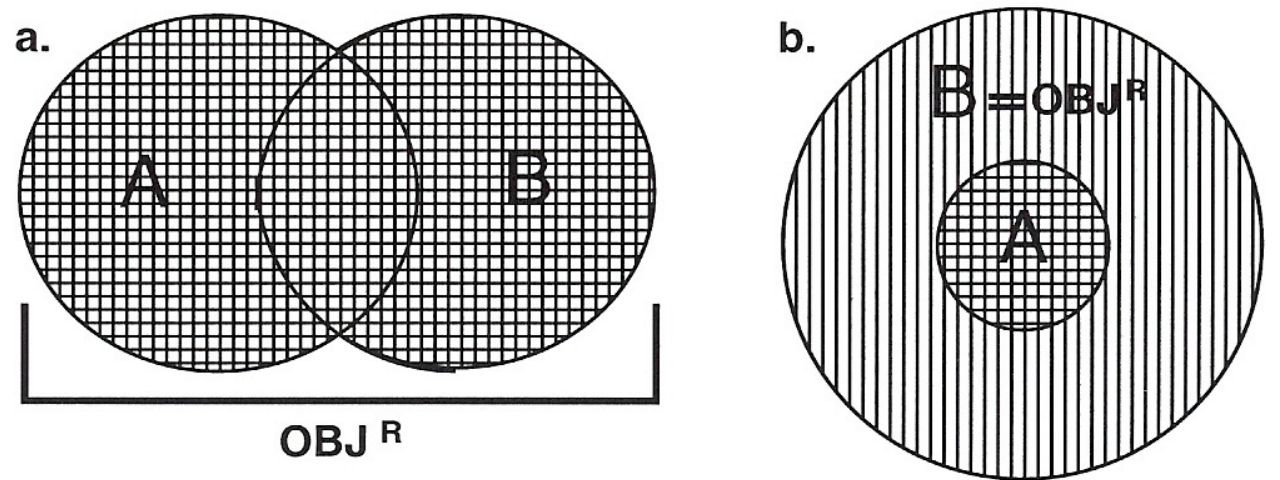

Figure 4 Basic naturalness has no FULL UNION for not totally distinct $A$ and $B$

Whereas it is true, at the level of basic naturalness, that FUR(A,A, $\left.{ }^{-\mathrm{R}}\right)$, as in

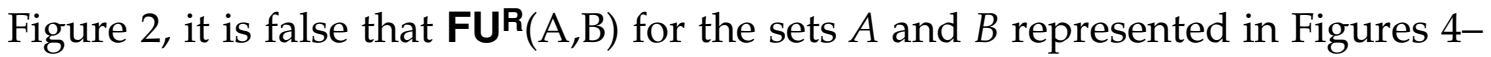
a,b. As for Figure 4-a, the relation between $A$ and $B$ lacks a name. In standard set theory it lacks one, presumably, because it is mathematically uninteresting. In basic NST it lacks one because no relation is seen there. Only in strict NST does it deserve a name ("full union"), because it corresponds to the metalogical relation of subcontrariety, which is relevant in predicate calculus. And as regards Figure 4-b, all that is involved is the relation of proper inclusion of $A$ in $B$. The relation FUR thus delivers truth, in basic natural set theory, for the natural sets $A$ and $B$ only when $B,{ }^{-R}={ }_{v}$ A or vice versa - that is, when the one is the restricted complement of the other, as illustrated in Figure 2. And since we already have a term for both that relation and that function, the notion of FULL UNION is not needed in natural set theory.

\subsection{Consequences for (meta)logical relations and functions}

The set-theoretic functions and relations can be translated into functions and relations of logic and metalogic. As one reads from Figure 1, the set-theoretic RELATIONS translate into metalogical relations. This is achieved by taking the valuation spaces of L-propositions $P$ and $Q, / P /$ and /Q/, respectively, as the arguments of the relations and by making the universe of all possible situations $\mathbf{U}$ stand for OBJ. The set-theoretic relations also translate into the standard operators of quantification in the object-language $\mathbf{L}_{\mathbf{L}}$ if $[[\mathrm{F}]]$ and $[[\mathrm{G}]]$ are the arguments of the relation. The set-theoretic FUNCTIONS are translated into propositional operators (truth functions) in the object-language $\mathbf{L}_{\mathbf{L}}$ again by taking / P/ and /Q/ as arguments and by making the universe of all possible situations $\mathbf{U}$ stand for $\mathbf{O B J}$. 
For standard and traditional Aristotelian-Boethian predicate calculus, we distinguish between the sentence types $\mathbf{I}$ and $\mathbf{A}$, with their external $(\neg)$ and internal $(*)$ negations:

I Some $F$ is $G$

A All $F$ is $G$

ᄀ $N$ No $F$ is $G$

$\neg A$ Not all $F$ is $G$ or equivalently or equivalently or equivalently or equivalently $\neg A^{*}$ Not all $F$ is not-G

$\neg$ ᄀ $^{*} \quad$ No $F$ is not-G

$A^{*} \quad$ All $F$ is not-G

$I^{*}$ Some $F$ is not-G

Likewise for the sentence schemata in propositional calculus, where OR stands for $P \vee Q$, AND for $P \wedge Q, O R^{\star}$ for $\neg P \vee \neg Q$, and $A N D^{\star}$ for $\neg P \wedge \neg Q$ :

\begin{tabular}{|c|c|c|c|c|}
\hline OR & $P \vee Q$ & or equivalently & $\neg \mathrm{AND}^{*}$ & $\neg(\neg P \wedge \neg Q)$ \\
\hline AND & $P \wedge Q$ & or equivalently & $\neg \mathrm{OR}^{\star}$ & $\neg(\neg P \vee \neg Q)$ \\
\hline$\neg \mathrm{OR}$ & $\neg(P \vee Q)$ & or equivalently & AND* & $\neg P \wedge \neg Q$ \\
\hline$\neg$ AND & $\neg(P \wedge Q)$ & or equivalently & OR* & $\neg P \vee \neg Q$ \\
\hline
\end{tabular}

Now the VS models shown in Figure 5-a,b can be set up for the sentence schemata in predicate and propositional calculus, respectively (space 4 in Figure $5-a$ is reserved for those situations in which the extension of the Fpredicate is null):
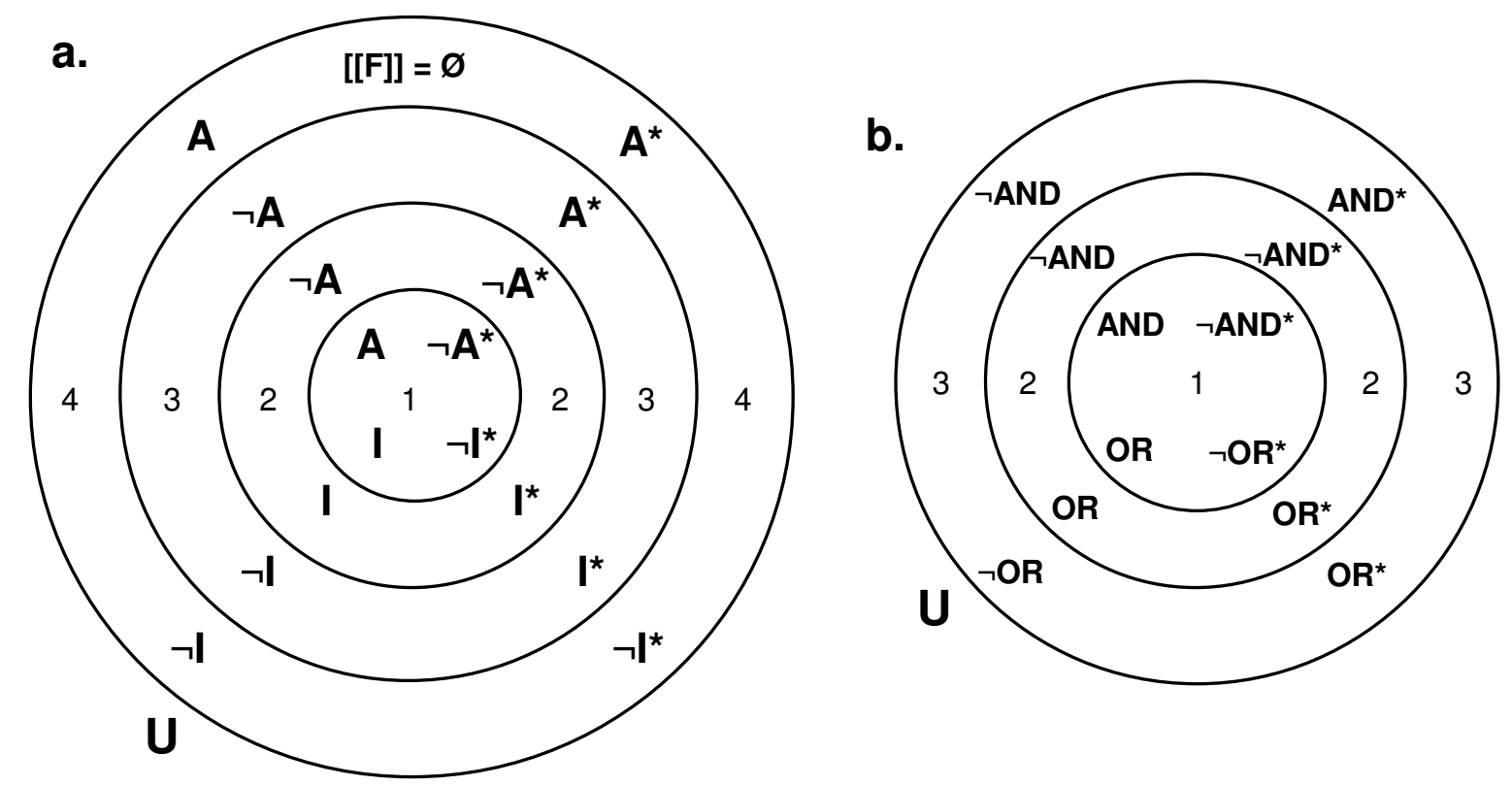

Figure 5 VS-models for (a) standard modern predicate calculus and

(b) propositional calculus 
Under the conditions imposed by NST, COMPLEMENT is modified into RESTRICTED COMPLEMENT $\left(X^{-}{ }^{-}\right)$, as defined in (6a), giving rise to a (presupposition-preserving) RESTRICTED NEGATION $(\sim)$. If $\mathbf{U}$ is restricted to the spaces 1,2 , and 3 of Figure $5-a$, the predicate logic within $\mathbf{U R}$ is traditional Aristotelian-Boethian predicate logic, with the subaltern entailment $\mathbf{A}$ I, - I, as shown in Figure 6. This predicate logic is then seen to be fully isomorphic with propositional logic. We consider both traditional Aristotelian-Boethian predicate logic and standard propositional logic to represent STRICT natural logic. BASIC natural logic is represented by the Hamilton-Jespersen system shown in Figure 9 and the basic precursor of standard propositional logic shown in Figure 11 below.

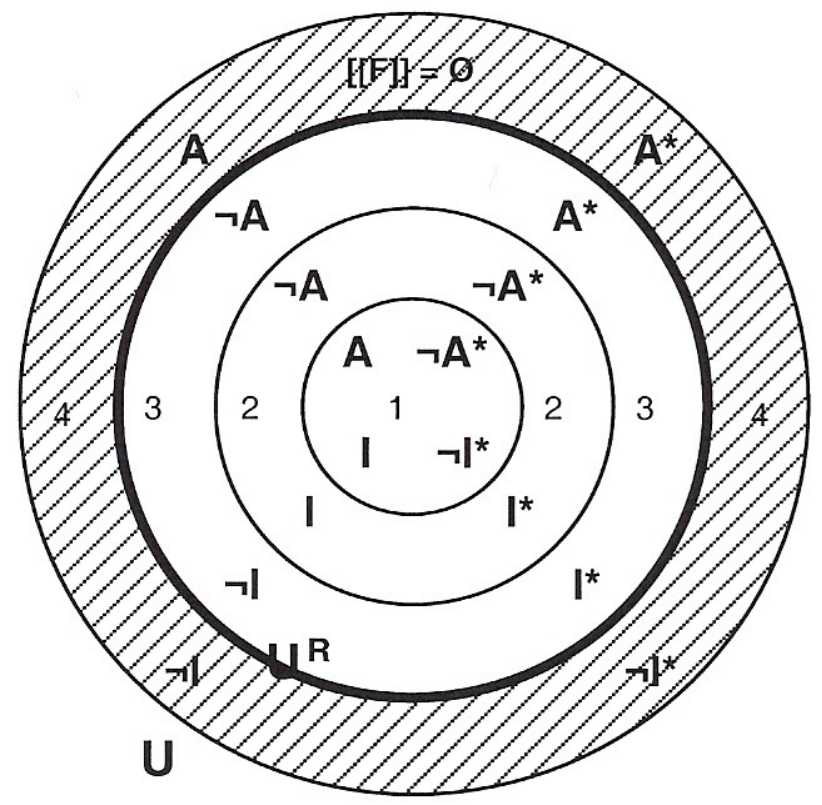

Figure 6 VS-model for predicate logic with $\mathbf{U R}^{\mathbf{R}}$ restricted to situations where $[[\mathrm{F}]] \bullet \varnothing$

PNST-3 excludes double negation. ${ }^{2}$ Double logically functional negation does occur, but only in culturally well-developed speech. Treble logically functional negation is rare and confusing. Quadruple logically functional negation is out of the question.

At the level of basic naturalness, DISJUNCTION is restricted to nonnull valuation spaces that do not intersect. CONJUNCTION is reduced to partial intersection and is defined only over actually intersecting sets of situations (valuation spaces) /P/ and /Q/ such that neither /P/ nor /Q/ equals $\varnothing$ or UR,

\footnotetext{
2 Except, of course, when the negation is copied for the functional purpose of reinforcement, as in the Cockney sentence 'E's never been no good to no woman, not never.
} 
as in Figure 3-b. (SUBTRACTION plays no role in propositional logic; if it did it would be restricted to valuation spaces $/ P /$ and $/ Q /$ such that $/ P / \subset / Q /$.)

As regards the relations, one remembers that the standard metalogical relations of EQUIVALENCE ( $\equiv)$, ENTAILMENT $(\mathrm{I},-)$, CONTRADICTION (CD), CONTRARIETY (C) and SUBCONTRARIETY (SC) are expressible as standard settheoretic relations in terms of valuation spaces, as shown in (1). Natural set theory now imposes restrictions on these standard metalogical relations. First, EQUIVALENCE has to go: It does not exist in natural set theory as a metalogical relation. Yet, as with the identification of sets in general, there is a fully natural COGNITIVE OPERATION OF IDENTIFICATION, which takes two sentences that have had different interpretative histories and identifies them at some level of understanding. For example, one may say that Jack lives in London is equivalent to Dr. Smith lives in London provided the expressions Jack and Dr. Smith refer to the same person. Likewise, when we say that, in basic natural set theory, Some $F$ is $G$ is equivalent with Some $F$ is not-G, what we mean is that they have been identified at some level of theoretical interpretation. Equivalence is, therefore, reinterpreted as identification.

Then, ENTAILMENT of $(1 \mathrm{~b})$, corresponding to inclusion as defined in standard terms in (7c) and redefined in terms of NST in (4), is a (basic and strict) natural relation, provided it is restricted to natural sets and to PROPER INCLUSION. The violently counterintuitive notion that a necessary falsehood $F$ entails any proposition - "ex falso per se ad quodlibet" - has been eliminated, because /F/ $={ }_{\mathrm{V}} \varnothing$, and $\varnothing$ is not a natural set. Likewise, the equally violently counterintuitive notion that a necessary truth $\mathrm{T}$ is entailed by any proposition - "verum per se ex quolibet" - has been eliminated, because $/ T /=_{\mathrm{V}} \mathbf{U}$, and $\mathbf{U}$ is not a natural set. The concept of naturalness introduced here further restricts the entailment relation in that identity of /P/ and /Q/ is now also excluded, which rules out the highly counterintuitive notion of self-entailment.

Entailments following from the theorem ("inference rule") of ADDITION have not been eliminated. Addition is the theorem saying that for any L-propositions $P$ and $Q$ it is always so that $P I,-P \vee Q$. Although this theorem is valid in standard propositional calculus, it seems clear that it should be qualified as nonnatural, since natural speakers will not agree that, for example, Sandy is married entails Sandy is married or today is Sunday. Yet NST fails to eliminate it. In standard terms, $/ P \vee Q /{ }_{v}=/ P / \cup / Q /$ and because $/ P / \subset(/ P / \cup / Q /)$, one must accept that $P I,-P \vee Q$. NST does not help here, because, even if $P$ and $Q$ are contraries, so that /P/ and /Q/ are totally distinct, as in Sandy is married or she died last year, it is still so that $/ P / \subset\left(/ P / \cup^{B N} / Q /\right)$, so that, in NST terms, $\mathrm{Pl},-\mathrm{P}$ or $\mathrm{Q}$. In other words, even for a necessarily exclusive OR, the entailment schema of addition remains intact.

To eliminate addition as a generally valid entailment schema, the notion of entailment must be sharpened in such a way that it requires not only that truth be preserved, but also that its preservation be determined by the specific 
linguistic meaning of the entailing L-proposition. This latter condition is not satisfied in cases of addition, as there is nothing in the specific meaning of any arbitrary $P$ that causes preservation of truth for $P$ or $Q, Q$ being equally arbitrary. Not so for the inference rule known as SIMPLIFICATION, which says that $P$ and $Q$ entails both $P$ and $Q$, since here it is the meaning of and that causes the entailments. Nothing much thus remains of addition.

All this taken together removes a great deal of counterintuitive excess baggage and, in fact, restricts the entailment relation to semantically motivated entailment, precisely as is wanted. We use the term NATURAL ENTAILMENT for the entailment relation as restricted by NST and by the stipulation that entailment is meaning-driven. It would seem that the notion of natural entailment properly delimits the class of entailments felt to be natural by native speakers, and hence empirically observable or measurable as psychologically valid data.

CONTRADICTION has been slimmed down to a contextually restricted UR, created by presuppositional restrictions on the possible situations in any discourse at hand. CONTRARIETY is the only relation that can stand unmodified, apart from the restriction of the VSs at issue to natural sets of possible situations. Finally, SUBCONTRARIETY has disappeared from the basic natural system, as it involves the relation of full union, which has been ruled out. Yet it reappears in the strict natural system of metalogical relations, though not without some considerable cognitive effort. ${ }^{3}$

This leaves the following list of (basically and strictly) natural metalogical relations:

(9) For all L-propositions $P$ and $Q$ such that /P/ and / $/$ are natural sets:
a. $\mathrm{CD}(\mathrm{P}, \mathrm{Q})$
iff $/ P / \cup / Q /=_{V} U^{R}$ and $/ P / \cap / Q /={ }_{V} \varnothing$ (or: $/ P /{ }_{v}=/ Q /,-R_{\text {) }}$
b. $C(P, Q)$ iff $/ \mathrm{P} / \cap / \mathrm{Q} /=_{\mathrm{V}} \varnothing$
c. $P I,-I,(=) Q$ iff $/ P / \subset / Q /$ (in virtue of the meanings of $P$ and $Q$ ).

\section{Problems with the NST-constrained system of logic}

So far, no great difficulties have been encountered. The difficulties start when one looks at the object-logical operators all, some, not, and, and or and the way they interact. As regards the operators of quantification, the only natural relations that can hold between sets $A$ and $B$, namely mutual exclusion, partial intersection, and proper inclusion, as shown in Figure 3, look as if they are directly reflected in the quantifiers no, some, and all, respectively. Likewise for

3 Aristotle, with all his logical acument failed to identify it (it was developed by his commentators), and beginning logic students, who still have to rely on their natural intuitions, tend to find subcontrariety very hard to grasp, as logic teachers know well. 
the propositional truth-functional operators, which look as if they are the direct reflections of the corresponding set-theoretic functions as redefined under NST. Yet when one tries to build a LOGICAL system - that is a system which maintains consistency - on the basis of these parallels between natural sets on the one hand and logical relations and functions on the other, one finds that there are complications.

\subsection{The NST-constrained system of basic natural quantification}

We start with predicate calculus. The predicate-calculus system within the constraints of NST at the level of basic naturalness as set out above turns out to be identical with the system that was defended by the nineteenth-century Edinburgh philosopher Sir William Rowan Hamilton (Hamilton 1860) and defended in a fierce polemic with the London logician Augustus De Morgan. The same system, by and large, was again proposed in the twentieth century in Jespersen (1917). Perhaps because Hamilton lost his war with De Morgan, and perhaps also because of the unique prestige of modern logic, Hamilton's system was never analysed in detail. Yet, given its undoubted intuitive appeal, it deserves a more precise analysis in the context of an investigation of natural cognition. The more so because Jespersen, who possessed a finely tuned intuition as regards linguistic matters but had no logical knowledge, came up with what looks very much like the same system for predicate logic (Jespersen 1917: 85-92).

We will look at this logic from the point of view of basic natural set theory, which allows us to be more precise than the Hamilton-Jespersen system, which is not in every way as explicit as one would wish. The logic is determined by three quantifiers: NO meaning 'null', SOME meaning 'partial' (henceforth $\mathrm{SOME}_{\mathrm{exc}}$ ) and equivalent to SOME $\mathrm{exc} \mathrm{NOT}$, and ALL meaning 'the complete set', plus bivalent negation within a restricted $\mathbf{U R}_{\mathbf{R}}$ that is, the negation written as $\sim$. Figure 7 shows the only possible basic natural relations between [[F]] and [[G]], just as the isomorphic Figure 3.

There is, of course, a question as regards existential import. Under a strictly extensional ontology, the system has existential import, and thus fails to cater for situations where $[[F]]=\varnothing$. Without a proper provision, this will make the system unsound. One such provision, which leaves the ontology strictly extensionalist, has been indicated above. It consists in posing the presuppositional condition that $[[\mathrm{F}]] \bullet \varnothing$. Since, however, the entailment of existence (existential generalization) is generated as a presupposition not only by the universal quantifier but by most ordinary lexical predicates in any natural language, the provision must be extended to cover those cases as well. The extension we take to be adequate, without arguing for it here as it is not directly germane to the main topic of this paper (but see Seuren in prep.), consists in the acceptance of a nonextensional ontology whereby any entailment 
of actual existence is determined by the lexical preconditions of the predicate $G$. The sets $[[F]]$ and $[[G]]$ are then by definition nonnull, as the very fact that $F$ or $G$ is brought to bear creates an intensional object or set of objects and hence a possible reference object or a possible set to quantify over. To obviate possible criticisms to the effect that undue existential import has not been reckoned with in the present analysis, we reserve a special "outer" space, marked * $[[\mathrm{F}]]^{*}$, in the Figures 8-a and 9-a for the set of situations not satisfying an existential precondition lexically imposed by any predicate $G$.

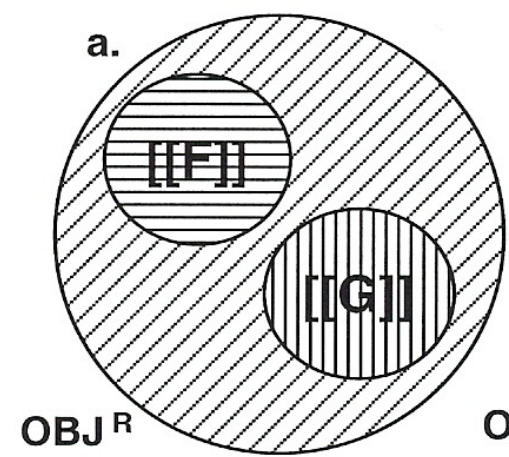

E: No $F$ is $G$ No $G$ is $F$ All $F$ is not-G All $G$ is not- $F$

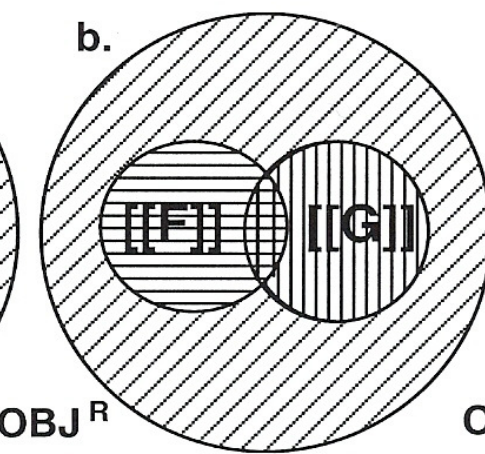

$I$ and $I^{*}$ : Some $F$ is $G$ Some $F$ is not $G$ Some $G$ is $F$ Some $G$ is not $F$

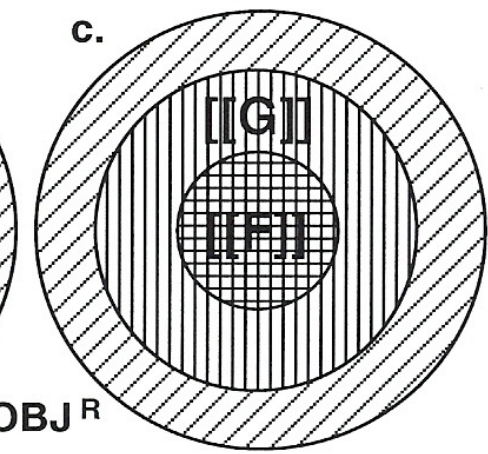

A: All $F$ is $G$ No $F$ is not-G Some $\mathrm{G}$ is $\mathrm{F}$ Some $G$ is not $F$

Figure 7 The possible basic natural relations of [[F]] and [[G]] and the naïve translations into predicate calculus terms

In this logic, the three quantifiers ALL, $\mathrm{SOME}_{\text {exc }}(-\mathrm{NOT})$, and NO denote the only three possible basic natural relations between two sets. From a cognitive point of view, therefore, their status is very strong. Yet despite their preferred status in cognition, there is a question as to the consistency, and hence the logical tenability, of the logic thus constrained. If the doubts regarding this logic prove justified, the conclusion is clear: Human cognition must yield to the demands of a consistent relation to the world and thus upgrade itself. Prototype is subservient to consistency.

The basic natural system can be looked at in two ways. First, one can follow the tradition and read the quantifier $\mathrm{NO}$ as a lexicalization of NOT-SOME $\mathrm{exc}$, with SOME excluding ALL - that is, $\mathrm{SOME}_{\mathrm{exc}}$ - and NOT expressing the restricted complement-selecting operator $\sim$. On this reading, the VS model of the basic natural system is as shown in Figure 8-a, leading to the Square of Figure 8-b. (I $\mathbf{I}_{\text {exc }}$ stands for Some exc $F$ is G; the asterisk indicates internal or "subsentential" negation, as in Some $F$ is not G. $)^{4}$

4 In the light of PNC-3 it is questionable whether spaces involving multiple negation, such as $/ \sim \mathbf{E}^{*} /$ or $/ \sim \mathbf{I}_{\mathrm{exc}}{ }^{*} /$ have cognitive reality. They have not been deleted from Figure 15.7 


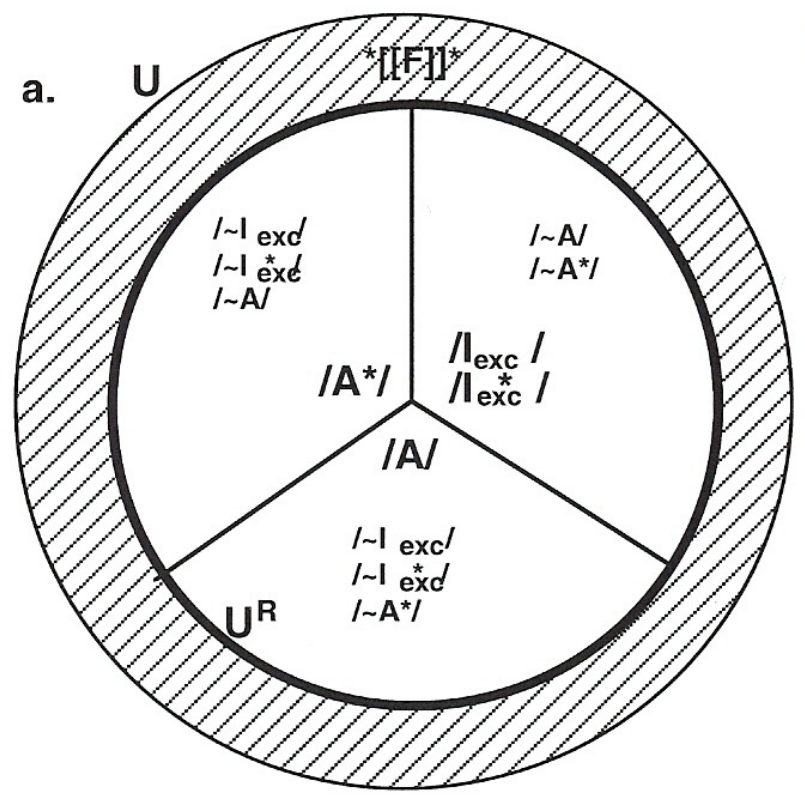

b.
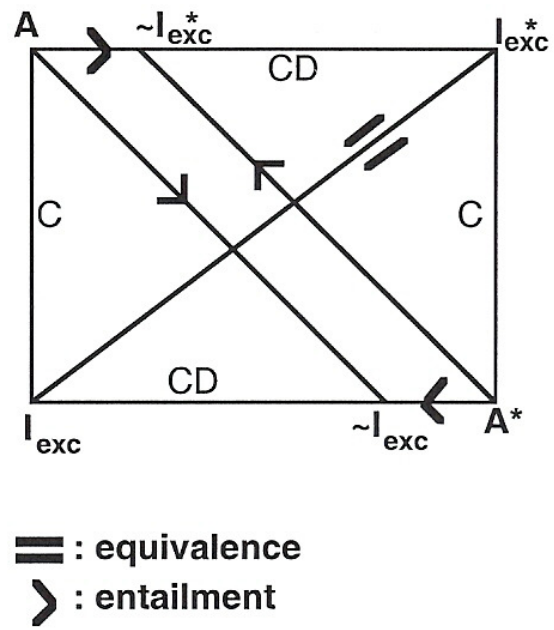

Figure 8 Basic natural quantificational logic with $\mathrm{NO}=$ NOT-SOME $\mathrm{exc}_{\mathrm{c}}$

Such a system, however, fails for a variety of reasons. For example, given the equivalence of $\mathbf{I}_{\text {exc }}\left(\right.$ Some $_{\text {exc }}$ is $G$ ) and $\mathbf{I}_{\text {exc, }}{ }^{*}$ (Some exc is not $G$ ), their negations should both be true in the set of situations forming the complement of $/ \mathbf{I}_{\text {exc }} \equiv$ $I_{\text {exc, }}{ }^{*} /$. But whether there are real flags or only virtual ones, linguistic understanding does not allow No flag is green and No flag is not green to be both true at the same time. Then, since in Figure 8-a, $/ \mathbf{I}_{\text {exc }} /,-$ comprises both $/ \mathbf{A}^{*} /$ and $/ \mathbf{A} /$, NO would have to mean 'either none or all', which is clearly not so. Moreover, All flags are green would have to entail No flag is green, which, of course, is semantically abhorrent. On this interpretation, therefore, the basic natural system is untenable as a logical system serving the purposes of natural language.

The system is improved when NO is not read as 'NOT-SOMEexc' but is taken to stand on its own as a separate quantifier equivalent to standard NOT-SOME (though "inclusive" SOME does not form part of this logic). A sentence of the type $\sim \mathbf{I}_{\text {exc }}$ is then no longer read as No $F$ is $G$, but rather as It is not so that (only) some $F$ is $G$, leaving open the possibility that either All $F$ is $G$ or No $F$ is $G$ is true. So let us symbolize No $F$ is $G$ as type $\mathbf{E}$, following Boethius, and distinguish $\mathbf{E}$ from $\sim \mathbf{I}_{\text {exc. }}$. This system is shown in Figure 9, where $/ \sim \mathbf{I}_{\text {exc }} /$ is the complement of $/ \mathbf{I}_{\text {exc }} /$ in $\mathbf{U R}$, but $/ \mathbf{E} /$ is only part of $/ \sim \mathbf{I}_{\text {exc }} /$, so that No $F$ is $G$ entails It is not so that some $F$ is $G$, but not vice versa. 

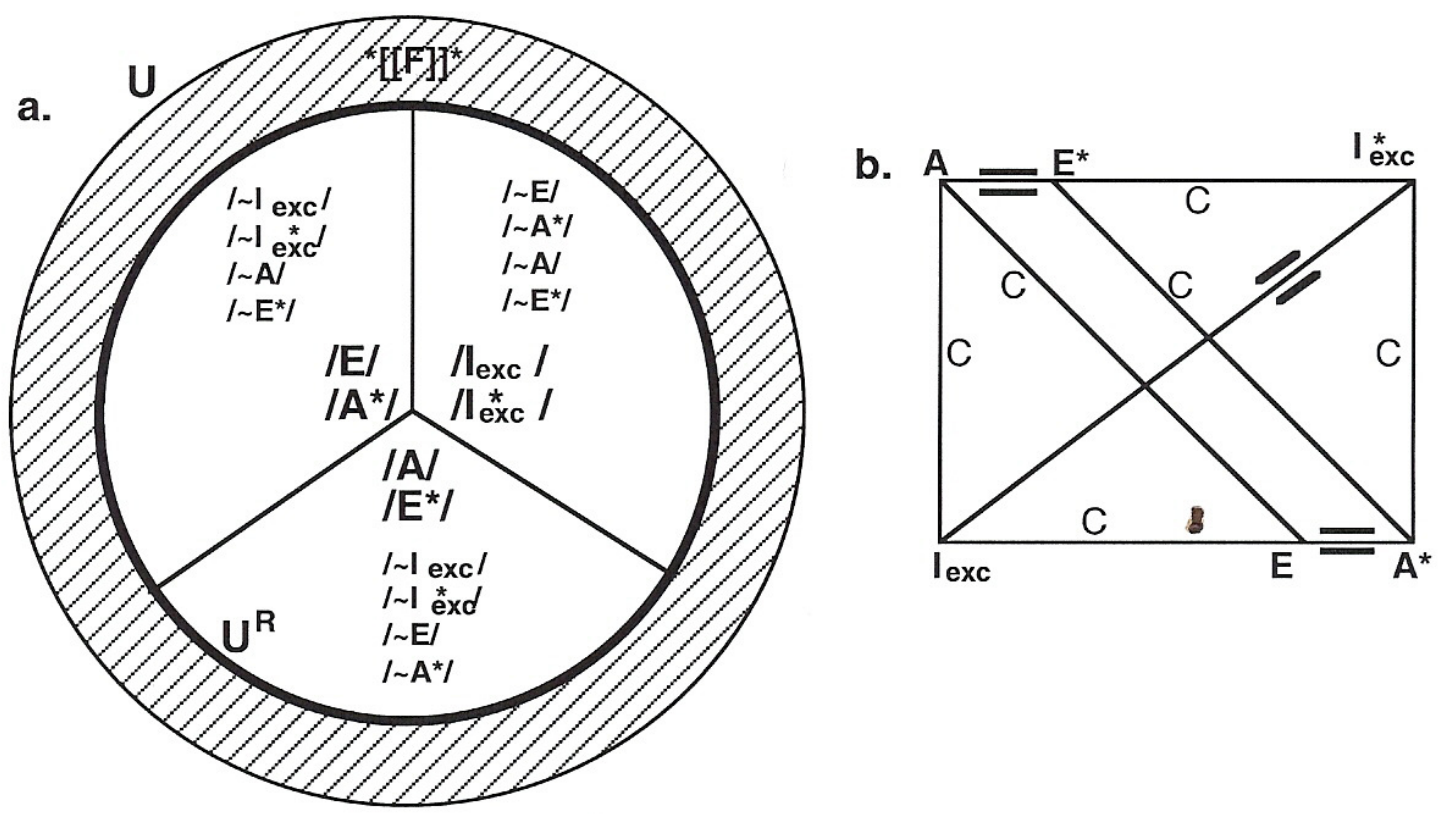

Figure 9 Basic natural quantificational logic with NO $\bullet$ NOT-SOME $E_{\text {exc }}$

Now the restricted universe $\mathbf{U R}^{\mathbf{R}}$ of situations where [[F]] satisfies the preconditions defined for the predicate $G$ is fully exhausted by $/ \mathbf{E} \equiv \mathbf{A}^{*} /, / \mathbf{I}_{\text {exc }} \equiv$ $\mathbf{I}_{\text {exc, }}{ }^{*} /$, and $/ \mathbf{A} \equiv \mathbf{E}^{*} /$, as shown in Figure $9-\mathrm{a}$, where $\mathbf{E} \equiv \mathbf{A}^{*}, \mathbf{I}_{\mathrm{exc}} \equiv \mathbf{I}_{\mathrm{exc}}{ }^{*}$, and $\mathbf{A}$ $\equiv \mathbf{E}^{\star}$ all stand to each other in the single relation of contrariety. Although this is an improvement, it still leaves open the possibility of both $\mathbf{I}_{\text {exc }}$ and $\mathbf{I}_{\text {exc, }}{ }^{*}$ being false simultaneously. In defence of the system one can say that it does not allow for $\mathbf{E}$ (No $F$ is $G$ ) and $\mathbf{E}^{\star}$ (No $F$ is not $G$ ) to be true simultaneously, which is in accordance with natural intuition, but then one must be resigned to the counterintuitive result that No $F$ is $G$ is not synonymous with Not-some exc $F$ is $G$. Now, in cases where All $F$ is $G$ is true - that is, for all situations in / A/ - No F is $G$ is false whereas Not some $F$ is $G$ is true, because it is still so, under this interpretation, that $\mathbf{A}$ l, $-\sim \mathbf{I}_{\mathrm{exc}}$, which is now to be read as All $F$ is $G$ entails $I t$ is not the case that (only) some $F$ is $G$.

This would be all right - apart from the question of existential import - if some meant 'only some but not all', but some is also naturally used in the sense of 'at least some, perhaps all'. A speaker can vouch for the truth of Some F is G when only part of $[[\mathrm{F}]]$ has been checked and so far all elements in [[F]] satisfy the conditions of the predicate $G$. The corresponding mental model will then, for the time being, look like Figure 7-b. But as the checking proceeds and the elements in [[F]] keep satisfying $G$, [[F]] will move further and further into [[G]], until, in the end, [[F]] may be totally included in [[G]], as in Figure 7-c. It will not do, of course, to say that the original Some $F$ is $G$ becomes false the moment the last element of $[[F]]$ is checked and found to satisfy $G$, since that would turn a truth into a falsehood during the same discourse without anything changing in 
the state of affairs $s$ but only as a result of increased knowledge of $s$. Consistency of discourse demands that both the existentially quantified and the universally quantified statements are true in such cases. Analogously, as long as checking fails to yield an element in [[F]] that satisfies $G$, Some $F$ is not-G will remain true but there will be no warrant for Some $F$ is $G$. And when this trend continues so that, in the end, no element in $[[F]]$ is found that satisfies $G$, the statement Some $F$ is not-G will still be true and Some $F$ is $G$ can be truthfully denied as No $F$ is $G$, without having to revise any truth established earlier.

In the second interpretation, the basic natural system of quantification thus labors under a lack of consistency through discourse and the counterintuitive difference between no and not-some exc, but otherwise the system shown in Figure 9 seems to be all right. WE THEREFORE TAKE IT THAT THE BASIC NATURAL SYSTEM OF PREDICATE LOGIC IS THE SYSTEM SHOWN IN FIGURE 9, WHERE NO •

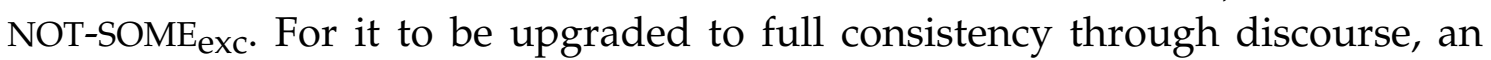
entailment from All $F$ is $G$ to Some $F$ is $G$ must be allowed - that is, the famous SUBALTERN entailment schema $\mathbf{A}$ I, - I, which has caused so much trouble in the history of logic. The introduction of the subaltern entailment schema amounts to an upgrading from basic to strict naturalness.

This conclusion is to some extent disconcerting, in that it amounts to saying that the basic natural set-theoretic relations of mutual exclusion, partial intersection, and proper inclusion as expressed by the quantifiers no, all, and some $_{\text {exc }}$, respectively, fail to guarantee consistency through discourse and thus form a system which is, in fact, logically inadequate. Had it not been for Aristotle, this would be grist to the modern logician's mill, as it has, for some time now, been customary among logicians to condemn natural language for being logically unreliable. Aristotle, however, made the world see that, on reflection, one must concede that $\mathbf{A}$-type sentences entail the corresponding Itype sentences, thereby removing the main blemish in the basic natural NSTconstrained system of predicate logic. Needless to say, the Aristotelian system conquered the world, until it was replaced, a century ago, with the modern, highly constructed, system of predicate logic often referred to as the Russellian system, which, ironically, banished again the subaltern entailment from A-type to I-type sentences so as to get rid of undue existential import. It does seem, however, that the problem of undue existential import can be solved by other means, so that a fully sound logic of natural language and cognition can be envisaged that is less far removed from natural intuitions and more Aristotelian in outlook.

\subsection{The NST-constrained system of propositional logic}

Is there an analog in propositional logic to the basic natural system of quantification? To a large extent there is, but there are also differences. We recall from Figure 1 that, unlike the quantifiers, which express relations 
between sets of objects, the propositional operators are functions mapping $n-$ tuples of VSs on VSs. They become predicates when predicated of the actual situation $s_{a c t}$, as shown in (10)-(12) below.

NEGATION is not immediately problematic (though, when taken in all its aspects, it is perhaps the most complex of all the functions). We define basic natural negation as follows ( $P$ ranges over contextually well-anchored and situationally well-keyed L-propositions):

(10) $\left[[\sim]=\left\{\mathrm{P} \mid s_{\text {act }} \in / \mathrm{P} /,-\mathrm{R}\right\}\right.$ (the extension of $\sim$ is the set of all L-propositions $\mathrm{P}$ such that the actual situation sact is a member of the restricted complement of $/ \mathrm{P} /$ )

CONJUNCTION $\left(\wedge^{\mathrm{BN}}\right)$ maps onto the BN-intersection of the VSs of the argument L-propositions $\left(\mathrm{P}^{+}\right.$stands for any number of semantically compatible L-propositions):

$$
\begin{aligned}
& {\left[\left[\wedge^{B N}\right]\right]=\left\{P^{+} \mid \text {sact } \in \cap^{B N} /^{+}\right\}} \\
& \text {(the extension of } \wedge \text { is the set of all sets of two or more propositions p, such that sact is } \\
& \text { a member of the } B N \text {-intersection of all } / P /^{+} \text {) }
\end{aligned}
$$

The argument L-propositions cannot be necessarily true or necessarily false (the VSs would equal $\mathbf{U}$ and $\varnothing$, respectively). Moreover, the conjunction as a whole cannot be necessarily true or necessarily false either. The fulfilment of this latter condition is ensured by PNST-2c, which stipulates that the VSs of the Lpropositions involved must partially intersect (that is, they must be semantically compatible). This ensures, first, that there is at least a chance of all the L-propositions united under conjunction to be true simultaneously. For if the VSs do not intersect, there is no possible situation in which the two Lpropositions are both true, which makes them contraries and their conjunction necessarily false. Since and is the standard discourse-increment function, this condition is, in fact, the same as the condition on any new increment to a discourse domain that it be compatible with all earlier increments, to avoid a reduction of the discourse domain's VS to zero.

Secondly, partial intersection ensures that the VS of one of the L-propositions involved does not include the VS of the other, which would make the conjunction necessarily true. The basic natural semantics of and thus selects the partial intersection in the basic natural intersection relation in Figure $3-b$, which is, therefore, defined only for L-propositions that neither include nor exclude each other in virtue of their meaning.

AND thus says that the actual situation $s_{a c t}$ is an element in the intersection of /P/ and /Q/ of Figure 10. The restricted universe $\mathbf{U R}^{\mathbf{R}}$ is defined by the 
condition that the operators in question do not yield radical falsity as a result of presupposition failure.

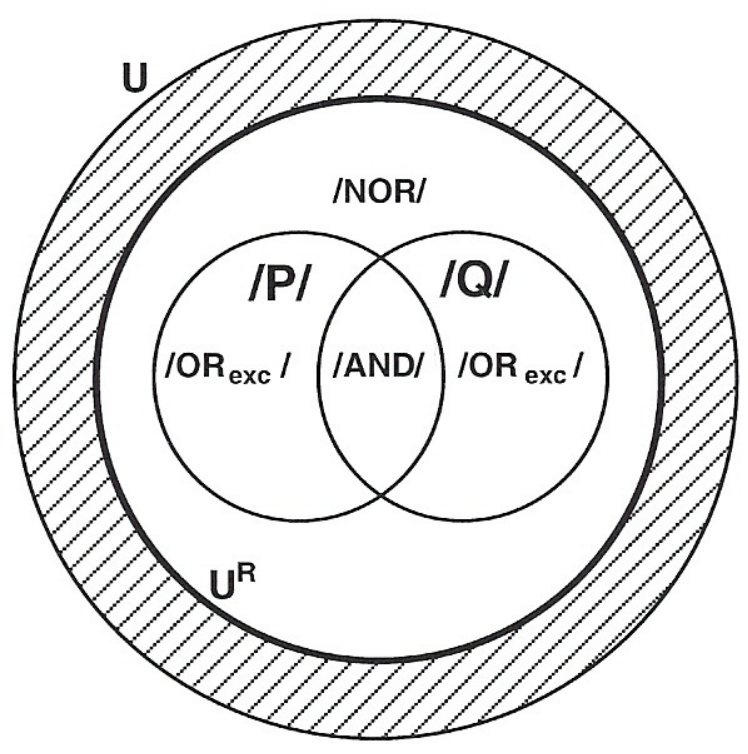

Figure 10 VSs for logically independent $P$ and $Q$ and their logical compositions

As regards DISJUNCTION $\left(\vee^{\mathrm{BN}}\right)$, the situation is more complex. We may take the linguistic disjunction operator or to map onto the BN-union of the VSs of the argument L-propositions, as defined in $(12)\left(\mathrm{P}^{+}\right.$stands for any number of Lpropositions):

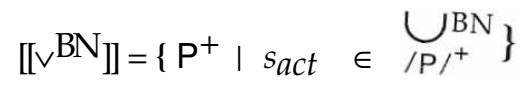

(the extension of $\vee$ is the set of all sets of two or more L-propositions P such that sact is a member of the BN-union of all $/ \mathrm{P} /{ }^{+}$)

Under the restrictions imposed on union by the criteria of basic naturalness, the component VSs must not only be natural sets (neither $\varnothing$ nor OBJ), but, according to PNST-2a, they must also be totally distinct - that is, without any intersection. However, natural language or is not restricted to contrary argument L-propositions: in a disjunction of the form $P$ or $Q, P$ and $Q$ must be allowed to be logically independent, so that their VSs, /P/ and /Q/, actually intersect, as in Figure 10. To satisfy PNST-2a, it is, therefore, necessary to reformulate $\mathrm{P}$ or $\mathrm{Q}$ for the purpose of linguistic interpretation at the level of basic naturalness in such a way as to ensure that the two arguments of the union function are totally distinct. 
This can be done in two ways. The least drastic way is to consider $P$ or $Q$ to be tacitly understood, in terms of $\wedge$ and $\vee$ and the restricted negation $\sim$, as:

$$
P \text { or (not- } P \text { and } Q) \text { (or: } P \vee(\sim P \wedge Q))
$$

In standard propositional logic, this is equivalent to $P \vee Q$, yet there is a difference in that in (13) the two disjuncts have totally distinct VSs, which, therefore, allow for the basic natural union operation. This interpretation, however, has the disadvantage that or is not exclusive, since if $s_{a c t} \in / \mathrm{P} /$, it may be the case that $s_{a c t} \in / P \wedge Q /$, so that type-AND L-propositions entail typeOR L-propositions. A more drastic reformulation is, therefore, needed to get the exclusive $\mathrm{OR}_{\mathrm{exc}}$ of basic natural propositional logic, corresponding to the mutually exclusive basic natural union of NST.

To get a proper $\mathrm{OR}_{\text {exc }}$ it is necessary to regard $\mathrm{P}$ or $\mathrm{Q}$ to be tacitly understood as (14), which is not equivalent to standard $P \vee Q: 5$

$$
\text { ( } P \text { and not- } \mathrm{Q}) \text { or (not- } \mathrm{P} \text { and } \mathrm{Q})(\text { or: }(\mathrm{P} \wedge \sim \mathrm{Q}) \vee(\sim P \wedge \mathrm{Q}))
$$

The disjunction operator $\mathrm{OR}_{\mathrm{exc}}$ now selects the union of /P/ and /Q/ in Figure 10, minus their intersection. This does account for the intuition that natural language or is exclusive, excluding cases where both $P$ and $Q$ are true.

5 The tacit understanding as formulated in (14) also accounts for the unnaturalness of a disjunction where one disjunct entails the other as in

(i) !The man is dead or he has been killed.

If this is tacitly understood as 'either the man is dead and he has not been killed or the man is not dead and he has been killed', then the second disjunct is necessarily false owing to the contrariety of 'the man is not dead' and 'he has been killed'. 

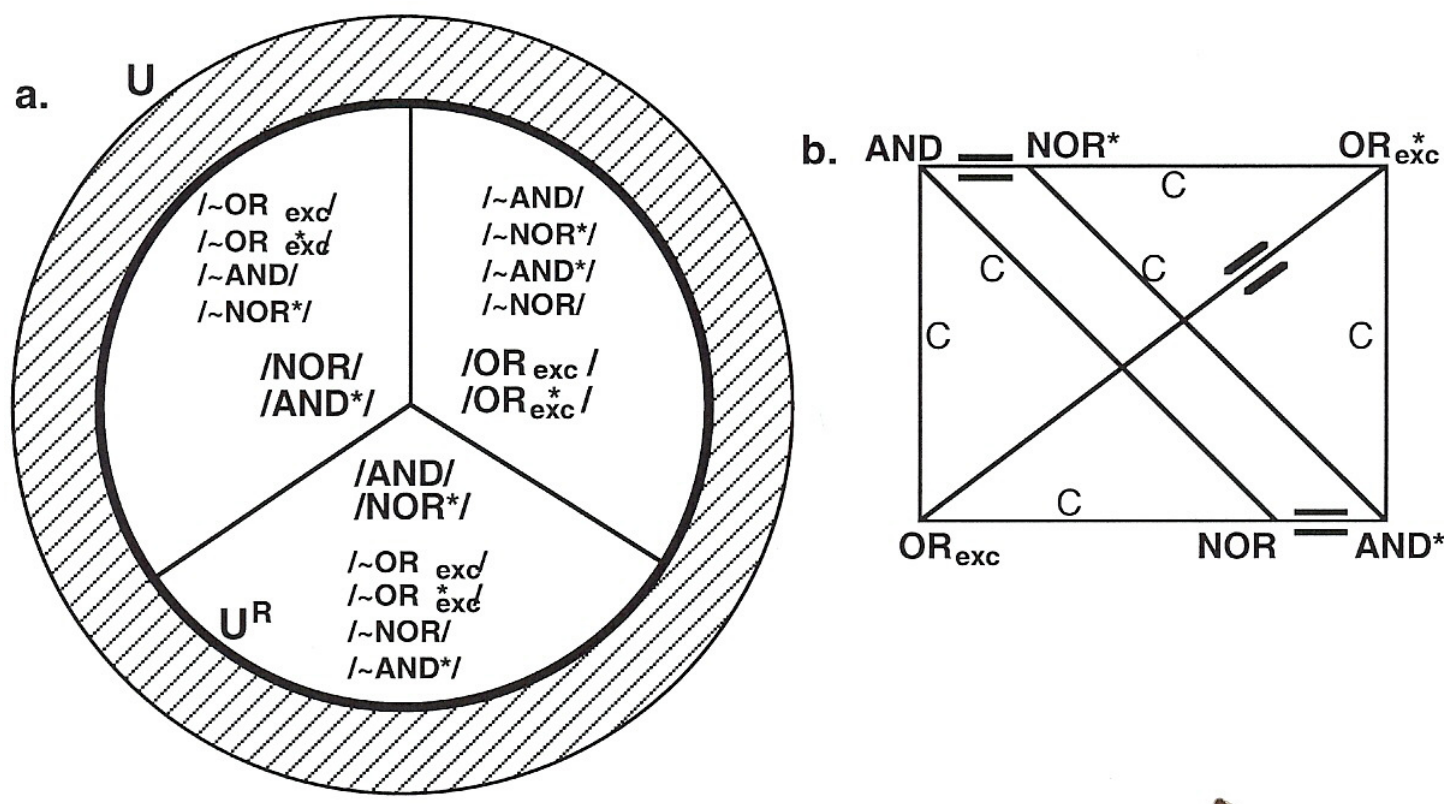

Figure 11 Basic natural analog for propositional logic with NOR $\bullet \sim$ OR

But how does this work out for the logic? In the reading (14), / AND / and /OR $\mathbf{R}_{\text {exc }}$ / are mutually exclusive and together form standard /OR/. The only space not covered in $\mathbf{U} \mathbf{R}$ is the restricted complement of standard / OR/ - that is, /OR $/^{\mathrm{R}}$, - normally denoted by the linguistic operator neither $a$... nor $b$, also realized as not ... a or $b$, and here called NOR. ${ }^{6}$ One notes that NOR cannot be the negation of the exclusive $\mathbf{O R}_{\mathrm{exc}}$ of this system, since the complement of /OR $\mathbf{O R x c}_{\text {ex }} /$ /OR $\mathbf{R}_{\text {exc }} /$, , comprises / AND/, and neither $P$ nor $Q$, or not $P$ or $Q$, clearly means not- $P$ and not $Q$, excluding $P$ and $Q$. Therefore, just as English no corresponds to standard NOT-SOME and not to basic natural NOT$\mathrm{SOME}_{\mathrm{exc}}$, English nor corresponds to standard (but discourse-restricted) NOT-OR and not to basic natural NOT-OR exc. Consequently, NOR is equivalent, in this system, with AND*, as shown in Figure 11.

In this basic natural system, $\mathbf{O R}_{\mathbf{e x c}}$ and $\mathbf{O R}_{\mathrm{exc}}{ }^{*}$ are equivalent, both being tacitly understood as specified in (14). Given the fact that $\mathbf{O R}_{\text {exc }}$ is accounted for in the logic of Figure 11, and given the parallelism with the predicate-logic system of Figure 9, we take it that THE BASIC NATURAL SYSTEM OF

6 In Seuren (1974) it is argued that an L-propositional structure of the form and [not-P, notQ] - that is, an L-proposition of type AND* - is grammatically transformed into not [P or Q], or NOT-OR, by the rule of NEGATIVE RAISING. Likewise all ... not, or $\mathbf{A}^{*}$ is taken to be transformed into not-I. I still feel that this analysis has a great deal going for it, though I would now add that these two meaning-preserving instances of NEGATIVE RAISING imply a lexical specialization into NOR and NO, and not just the negation of $\mathrm{OR}_{\mathrm{exc}}$ and SOME exc, respectively. 
PROPOSITIONAL LOGIC IS THE SYSTEM SHOWN IN FIGURE 11, WITH NOR • NOTOR $_{\text {exc. }}{ }^{7}$

Empirical evidence for this point of view is derived, for example, from the fact that a sentence like (15a) is naturally and immediately interpreted as (15b), whereas (16a) is not at all naturally and immediately interpreted as (16b), even though, from a strictly logical point of view, both (15) and (16) merely instantiate De Morgan's Laws. This is a fact on which neither the Gricean maxims, nor indeed the whole of pragmatics, have anything to say:

(15)a. He doesn't like tea or coffee.

b. He doesn't like tea and he doesn't like coffee.

(16)a. He doesn't like tea and coffee.

b. He doesn't like tea or he doesn't like coffee.

Given the analysis given above, it is now easy to see why this should be so. Consider not ... tea or coffee in (15a) to be an instance of basic natural NOR (see note 6). Figure 11 shows that, in this reading, NOR is equivalent to AND*, both sharing the same VS. By contrast, not ... tea and coffee in (16a) realizes $\sim$ AND, the restricted negation of AND, whose VS comprises the combined VSs of NOR (三 $\left.\mathbf{A N D}^{*}\right)$ and $\mathbf{O R}_{\mathrm{exc}}\left(\equiv \mathbf{O} \mathbf{R}_{\mathrm{exc}}{ }^{*}\right)$. It follows not only that the interpretation of (16a) requires a great deal more computation than the interpretation of (15a), but also that this computation is to take place at the level of strict naturalness (or even at the standard constructed level), since the or of (16b) is not the exclusive or exc but the standard inclusive or. This latter fact follows from PNST-2a, which requires total distinctiness in the case of union (disjunction). Clearly, the restricted negations of he likes tea and he likes coffee are logically and semantically independent, so that the corresponding VSs intersect partially and thus exclude their being processed under the basic natural operation of union.

One sees that, despite the differences with the basic natural system of predicate logic with NO - NOT SOME exc shown in Figure 9, the eventually resulting system for propositional logic under the same principles turns out to be completely parallel. And, likewise in parallel with the basic natural system of quantification, there is the counterintuitive fact that natural language nor does not stand for the basic natural not-ORexc.

Moreover, there is, again, the problem of consistency through discourse. As has been widely observed, or is typically used in situations where the speaker is uncertain as to which of the disjuncts provides the correct answer to the question he or she is entertaining but where the speaker has concluded that either disjunct will do as a good enough answer. This conclusion is naturally

7 NOR $^{\star}, \sim$ NOR, and $\sim$ NOR $^{\star}$ are undefined at the level of basic naturalness, due to PNST-3. As before, however, they are still mentioned in Figure 11-a, because our interest is in the ultimate logical consequences of the system when it is pursued to the end. 
expressed by the auxiliary of epistemic necessity must (Kratzer 1979). In the course of an investigation, a speaker may say $(17 b)$, in response to the question (17a):

(17)a. How did the journalist know?

b. The journalist must have spoken to Ann or to Jeremy.

If it is then found out that the journalist had spoken to both Ann and Jeremy, it would be incorrect to say that the person who uttered (17b) during the investigation had been wrong, even if that speaker had failed to think of the possibility that the journalist had spoken to both Ann and Jeremy.

Therefore, in parallel with the basic natural system of quantification, consistency through discourse requires an entailment from AND to OR. This time it was the Stoic philosophers who, roughly a century after Aristotle, discovered this fact and upgraded the natural propositional logic of language and cognition to full consistency by introducing the entailment from AND to OR, thereby creating the INCLUSIVE OR of standard propositional calculus.

a.

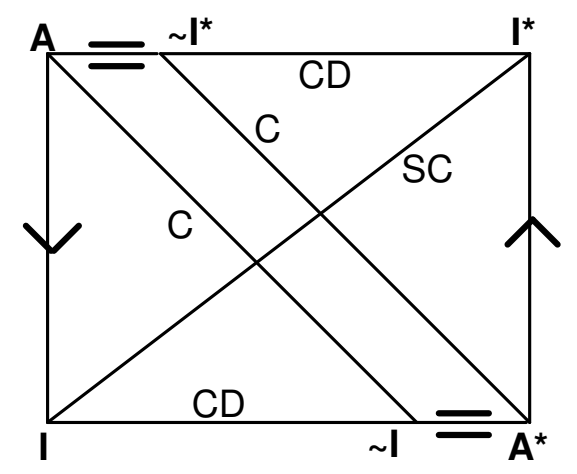

b.

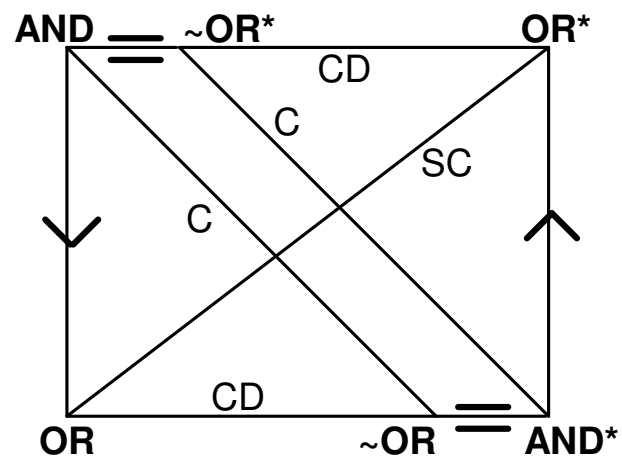

Figure 12 The upgraded strict natural squares for predicate and propositional logic, corresponding to the VS models in Figure 6 and 5-b, respectively

The parallelism of the two basic natural systems of predicate and propositional logic is thus maintained by the introduction of the parallel (subaltern) entailment schemata $\mathbf{A}$ I, - I and ANDI, - OR. The former results in strict natural predicate calculus, which is identical with traditional AristotelianBoethian predicate calculus and is shown in Figure 12-a. The latter results in strict natural propositional calculus, which is identical with standard propositional calculus and is shown in Figure 12-b. 


\section{Neither ${ }^{*}$ nand nor ${ }^{*}$ nall: NST predicts their absence}

We have reached a point where a question can be discussed which has recently attracted some attention. After it had been observed by Thomas Aquinas (see Horn 1989: 253), it was again observed more recently (Zwicky 1973; Horn 1972, 1989: 252-267; Levinson 2000: 69-71) that while a large majority of languages have single-morpheme expressions for NOT-SOME (such as English no), a singlemorpheme expression, say ${ }^{*}$ nall, for the complex negative predicate NOT-ALL or its presumed equivalent SOME-NOT has not so far been attested. Analogously, while many languages have a lexeme for not $(P$ or $Q)$ or equivalently not-P and not-Q (English neither ... nor), a lexeme corresponding to ${ }^{*}$ nand, for not( $P$ and $Q$ ) or equivalently not-P or not- $Q$ appears to be either nonexistent or at most extremely rare. ${ }^{8}$ The systematic absence of equivalents of *nall and *nand in the languages of the world appears to be matched by similar gaps in other lexical fields. Thus, whereas one finds lexicalized equivalents of epistemic NOTPOSSIBLE, lexicalizations of epistemic NOT-NECESSARY are nowhere to be found (lexicalized agentive NOT-NECESSARY, such as English unnecessary is widely attested). Likewise, NOT-CAUSE is never lexicalized, while NOT-ALLOW is frequently found in lexicalizations such as disallow, forbid or prohibit. Typically, predicates like necessary or cause show semantic characteristics that may lead one to think that they can or should be classified along with all and and: They all belong to an "all-yes" section of the lexicon, while possible and allow typically belong to the group of "perhaps-yes-perhaps-no" predicates, which also comprises the existential quantifier and the propositional connective or. The question is: Are these similarities reducible to a single principle, and if so, what is it? This question is interesting as it forces one to probe natural set theory in both the logic and the lexicalization processes of natural language.

Horn, Levinson and others seek an answer in the pragmatics of language use. Restricting themselves to *nand and *nall, they argue, in essence, as follows. Since or is normally exclusive for pragmatic reasons, excluding the case that both argument propositions are true, there appears to be no need left for an item like *nand which excludes the simultaneous truth of both argument propositions and may be taken to imply pragmatically that at least one of the argument propositions is true. Then, given the pragmatic equivalence of or and *nand, the item without the incorporated negation - that is, or - would be preferred on grounds of simplicity in the lexicon, so that *nand is ruled out. Similarly, since some often pragmatically implicates 'not-all', and can thus be taken to convey the intended meaning 'some but not all', it is assumed that there is no need left for a lexicalized form like *nall meaning 'not-all', which, in

8 Eric Pederson has informed me that Tamil does have a single lexeme meaning 'not and' or 'either one of the two or none'. Although this is an interesting and clearly relevant observation, it will require a great many more such observations to establish typological parity between lexemes for NEITHER...NOR on the one hand and NOT-AND on the other. 
standard logic, would be equivalent to 'some-not' and be taken to imply pragmatically 'only some'. In other words, there is no need for operators like *nand or *nall, since the pragmatically equivalent but cognitively and semantically simpler operators or and some are already available (Levinson 2000: 70). Therefore, the lexicalized expressions all, some and no will do for the quantifiers, and and, or and neither ... nor for the propositional operators.

For the authors who propose this explanation such a system is not a logical system but represents the way listeners construct a quantified mental model of a state of affairs described, on the presumption that the speaker has full and adequate knowledge of that state of affairs and has the intention to be as informative and helpful as possible - that is, that speakers will commit themselves to the maximum of what they know. The main criterion of such a system is not truth but INFORMATION VALUE ON THE PRESUMPTION OF FULL COOPERATIVITY AND COMPLETE KNOWLEDGE. It is not meant for the computation of solid entailments grounded in inescapable semantic properties, but for practical inferences. To say that two expressions are pragmatically equivalent then amounts to saying that they have the same information value on the presumption specified.

The question is, however, whether one may justifiably posit that linguistic lexicalizations depend on information value on the presumption of full cooperativity and complete knowledge. It would seem that such a position is unwarranted, since the use of language is not restricted to authoritative and maximally cooperative reporting on states of affairs fully known to the speaker. For one thing, as has been said, reporting often consists in informing the listener of WHAT HAS BEEN FOUND OUT SO FAR. When inspecting a population of children, a reporter may say that so far no, or so far some, or so far all, children have proved to be undernourished, without any commitment as to what is still to be found out. This point was made by Hoeksema, who first asks (Hoeksema 1999: 4) "If nall is not needed, due to the presence of some, then why is not all used at all?" and then observes (Hoeksema 1999: 5):

In contexts where the speaker has only partial knowledge, there is not even pragmatic equivalence. If I say that some of my students are gay, one should not infer immediately that not all my students are gay. Perhaps I am unaware of the sexual preferences of the remainder. But if $\mathbf{I}$ and $\mathbf{O}$ are often not even pragmatically equivalent, because the conditions for the Gricean implicatures are not met, then why should $\mathbf{O}$ be superfluous?

Since any system of interpretative cognitive model building for linguistic utterances depends primarily on pre-existing linguistic meanings, rather than on a presumption of full cooperativity and complete knowledge, it would seem that the pragmatics answer is not satisfactory on its own terms.

But there is an even more decisive argument. First we observe that the pragmatics answer described above depends on the assumptions (a) that traditional Aristotelian-Boethian predicate calculus and standard propositional 
logic form the correct logic for language (standard Russellian predicate logic has already tacitly been shunted), and (b) that natural lexicalization processes conform to the pragmatic implementation of that logic. In the perspective developed here, the latter assumption is replaced with the assumption that natural lexicalization processes conform to the primitive and inadequate basic natural logic of the Figures 9 and 11.

Horn (1989) recognizes that his pragmatic system reflects the Hamilton/Jespersen predicate logic - that is, the system shown in Figure 9 - but fails to mention that in that logic NO does not stand for NOT-SOME $E_{\text {exc }}$, and analogously for NOR, which does not stand for NOT-OR exc, NO and NOR being operators in their own right. There are thus, at the level of naturalness at which lexicalizations are deemed to take place, no single-morpheme expressions for NOT-SOME and NOT-OR. This, in effect, takes the bottom out of the question of why lexicalizations for NOT-ALL and NOT-AND are systematically absent, as it arose in the first place because NOT-SOME and NOT-OR were thought to lack counterparts for ALL and AND, respectively. In fact, however, given the lack of single-morpheme lexicalizations for NOT-SOME and NOT-OR, NST predicts the absence of such lexicalizations for NOT-ALL and NOT-AND, which in turn is valuable confirmation for the correctness of our reconstruction of basic natural logic as expressed in the Figures 9 and 11.

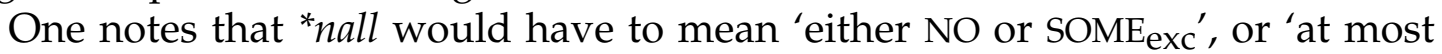

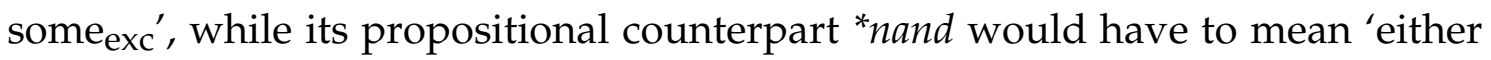
NOR or $\mathrm{OR}_{\text {exc' }}$, as does the Tamil connective mentioned in note 8. (Note that $\mathbf{I}^{*}$ and $\mathrm{OR}^{*}$ are unlikely to be lexicalized, for why should internal negation, being part of one or more arguments, be lexically incorporated?) *Nall would thus be defined in terms of a quantifier, $\mathrm{SOME}_{\mathrm{exc}}$, which does not occur in strict natural logic but does in basic natural logic, while no = NOT-SOME would be defined in terms of a quantifier, SOME, which does not occur in basic, but does occur in strict natural logic. And analogously for propositional logic: *nand would be defined in terms of an operator, $\mathrm{OR}_{\mathrm{exc}}$, which does not occur in strict natural logic but does in basic natural logic, while nor would be defined in terms of an operator, OR, which does not occur in basic natural, but does in strict natural logic.

The simplest answer would seem to be that NOT is never lexically incorporated into any operator of the logical system of which it is part, though it very often is into other lexical predicates. In many languages, including English, (the equivalent of) no, which lexicalizes the natural set-theoretic relation of mutual exclusion, clearly does contain an incorporated negation, but not in combination with a quantifier of the same logical system. Latin nullus, for example, is a combination of the negation word ne and unulus, the diminutive of unus (one), like the Dutch expression niet eentje ('not a little one'). Greek oudeis is literally 'not one', and so on. Such lexicalizations all deny the presence of even the smallest common element in the two sets involved. It is merely 
accidental that this coincides with the negation of existentially quantified Lpropositions - that is, with $\sim \mathbf{I}$ - in both standard modern and strict natural predicate calculus, but not in its basic natural counterpart. English no and its equivalents in many other languages thus appear not to lexicalize the meaning NOT-SOME but, rather, the absence of even the smallest common element in the two sets at issue - both linguistically and psychologically a plausible form of lexicalization, which, moreover, lends itself to being incorporated into a variety of sound systems of predicate logic. An analogous analysis applies to propositional logic.

Our close inspection of predicate and propositional logic from the point of view of cognitive naturalness sheds an interesting new light on Aristotle's and the Stoa's position in the history of logic and semantics. Whereas it is not totally unknown that the Stoics were sensitive to the role of discourse in natural language, in that they knew that utterances are, in principle, contextually anchored and referentially keyed, historians have so far been unaware of the fact that Aristotle's predicate logic as well as his notion of proposition, and also the Stoic creation of propositional logic, were all driven by the requirements imposed by the criterion of consistency through discourse. Significantly, where basic natural predicate and propositional logic fail to be discourse-proof and hence fail to be fully consistent, the Aristotelian and the Stoic upgradings of these branches of logic filled precisely the gaps that had to be filled for full consistency to be achieved, and they did so in a way that minimally intruded upon the basic natural systems. ${ }^{9}$

\section{References}

Barwise, Jon, and Robin Cooper (1981) Generalized quantifiers and natural language. Linguistics and Philosophy 4.2: 159-219.

Butterworth, Brian (1999) The Mathematical Brain. London: Macmillan.

De Morgan, Augustus (1847) Formal logic: or, the calculus of inference, necessary and probable. London: Taylor \& Walton.

Dehaene, Stanislas (1997) The number sense. New York: Oxford University Press.

${ }^{9}$ It is known from the history of logic that in ancient times these first upgradings, which already ran counter to natural intuitions, caused some uproar not only among academics but also among the general public, at least in Egyptian Alexandria, where the poet-librarian Callimachus wrote an epigram about the nonnaturalness of material implication, just introduced into logic by the Stoics: "Even the crows on the rooftops caw about the nature of conditionals" (Kneale \& Kneale 1962: 128). 
Dehaene, Stanislas (2005) Evolution of human cortical circuits for reading and arithmetic: The "neuronal recycling" hypothesis. In Stanislas Dehaene, Jean-René Duhamel, Marc D. Hauser, and Giacomo Rizzolatti (eds.), From monkey brain to human brain. A Fyssen Foundation symposium. Cambridge: MIT Press.

Dehaene, Stanislas, Véronique Izard, Pierre Pica, and Elisabeth Spelke (in press) Core knowledge of geometry in an Amazonian indigene group. Science.

Ginsburg, Herbert P., and Barbara S. Allardice (1984) Children's difficulties with school mathematics. In: Barbara Rogoff and Jean Lave (eds.), Everyday cognition: Its development in social context. Cambridge, Mass.: Harvard University Press, pp. 194-219.

Hamilton, William (1860) Lectures on Logic. Edinburgh: Blackwood \& Sons.

Hoeksema, Jack (1999) Blocking effects and polarity sensitivity. In J. Gerbrandy, M. Marx, M. de Rijke, and Y. Venema (eds.), JFAK. Essays Dedicated to Johan van Benthem on the Occasion of his 50th Birthday. Amsterdam: Amsterdam University Press (not available in hard copy) www.illc.uva.nl/j50/contribs/hoeksema/hoeksema.pdf

Horn, Larry R. (1989) A Natural History of Negation. Chicago/London: The University of Chicago Press.

Jespersen, Otto (1917) Negation in English and Other Languages. Det Kgl. Danske Videnskabernes Selskab, Historisk-filologiske Meddelelser I,5. Copenhagen: Andr. Fred. Høst \& søn.

Kneale, William, and Martha Kneale (1962) The Development of Logic. Oxford: Clarendon Press.

Kratzer, Angelika (1979) Conditional necessity and possibility. In Rainer Bäuerle, Urs Egli, and Arnim von Stechow (eds.), Semantics from Different Points of View. Berlin-Heidelberg-New York: Springer, pp. 117-147.

Levinson, Stephen C. (2000) Presumptive Meanings. The Theory of Generalized Conversational Implicature. Cambridge, Mass.: MIT Press.

Pica, Pierre, Cathy Lemer, Véronique Izard, and Stanislas Dehaene (2004) Exact and approximate arithmetic in an Amazonian indigene group. Science 306.(October 2004): 499-503.

Seuren, Pieter A.M. (1974) Negative's travels. In Pieter A.M. Seuren (ed.), Semantic Syntax. Oxford Readings in Philosophy. Oxford: Oxford University Press, pp. 183-208.

Seuren, Pieter A.M. (in prep.) Language and Logic in Cognition. 
138 Pieter A.M. Seuren

Van Fraassen, Bas (1971) Formal Semantics and Logic. New York-London: Macmillan.

Zwicky, Arnold (1973) Linguistics as chemistry: The substance theory of semantic primes. In Stephen Anderson \& Paul Kiparsky (eds.), A Festschrift for Morris Halle. New York: Holt \& Cy, pp. 467-485. 